NBER WORKING PAPER SERIES

\title{
NONLINEAR PRODUCTION NETWORKS WITH AN APPLICATION TO THE COVID-19 CRISIS
}

\author{
David Baqaee \\ Emmanuel Farhi \\ Working Paper 27281 \\ http://www.nber.org/papers/w27281
NATIONAL BUREAU OF ECONOMIC RESEARCH
1050 Massachusetts Avenue
Cambridge, MA 02138
May 2020

We thank Veronica De Falco, Sihwan Yang, and Stephanie Kestelman for excellent research assistance. We thank Natalie Bau for her comments. The views expressed herein are those of the authors and do not necessarily reflect the views of the National Bureau of Economic Research.

NBER working papers are circulated for discussion and comment purposes. They have not been peer-reviewed or been subject to the review by the NBER Board of Directors that accompanies official NBER publications.

(C) 2020 by David Baqaee and Emmanuel Farhi. All rights reserved. Short sections of text, not to exceed two paragraphs, may be quoted without explicit permission provided that full credit, including $(\odot$ notice, is given to the source. 
Nonlinear Production Networks with an Application to the Covid-19 Crisis

David Baqaee and Emmanuel Farhi

NBER Working Paper No. 27281

May 2020

JEL No. E0,E1,E2,E30

\begin{abstract}
$\underline{\text { ABSTRACT }}$
We study the effects of negative supply shocks and shocks to the composition of final demand on aggregate output in a disaggregated neoclassical model with multiple sectors, factors, and inputoutput linkages. We show how nonlinearities associated with complementarities in consumption and production amplify the effect of negative supply shocks by creating supply bottlenecks and disrupting supply chain networks. These nonlinearities are particularly potent when the shocks are more heterogeneous as the worst-affected sectors drag down the other sectors. Nonlinearities are strengthened when changes in preferences lead households to tilt the composition of their demand towards the crippled sectors directly and indirectly through their supply chains. And nonlinearities are further intensified when factors cannot easily be reallocated across sectors to reinforce weak links. A quantitative investigation suggests that nonlinearities may amplify the impact of the Covid-19 shock by between 10\\%-100\\%, depending on the horizon of analysis and the exact size of the shocks.
\end{abstract}

\author{
David Baqaee \\ Department of Economics \\ University of California at Los Angeles \\ Bunche Hall \\ Los Angeles, CA 90095 \\ and CEPR \\ and also NBER \\ baqaee@econ.ucla.edu \\ Emmanuel Farhi \\ Harvard University \\ Department of Economics \\ Littauer Center \\ Cambridge, MA 02138 \\ and CEPR \\ and also NBER \\ emmanuel.farhi@gmail.com
}




\section{Introduction}

The outbreak of the Covid-19 pandemic has focused the world's attention on nonlinearities. Thousands of pages have been written about the importance of slowing the spread of the disease, an effort dubbed flattening the curve, in order to avoid overwhelming the healthcare system. In this paper, we focus on a different kind of nonlinearity: the nonlinear response of aggregate output to the variety of supply and demand shocks that came with the pandemic. That is, we focus on how these shocks interact and how their effects are amplified at the macro level. Much like the spread of the disease itself, understanding the disease's effect on the macroeconomy also requires grappling with nonlinearities.

We use a general disaggregated model and aggregate up. We allow for an arbitrary number of sectors and factors as well as arbitrary input-output linkages and elasticities of substitution in production and in consumption. We analyze how the various micro shocks induced by the pandemic affect macro aggregates such as output and welfare. The nonlinear responses of these macro aggregates are shaped by the disaggregated structure of the economy.

We model the outbreak of the pandemic as a combination of exogenous shocks to the quantity of factors supplied, the productivity of producers, and the composition of final demand by consumers across sectors. One the one hand, the epidemic reduces the quantity of factors available, as workers withdraw from the labor force due to lock-downs or a reduced willingness to work. On the other hand, the epidemic reduces productivity of firms by forcing them to change their production plans. Finally, the epidemic changes the composition of final demand across goods and sectors as households rebalance their expenditures even in the absence of price changes because their preferences have changed or because regulations have been introduced. ${ }^{1}$

To frame the discussion, we rely on Hulten's theorem (Hulten, 1978). The theorem says that to a first-order approximation, the response of real GDP $\Delta \log Y$ to productivity shocks $\Delta \log A_{i}$, factor supply shocks $\Delta \log L_{f}$, and shocks to the composition of final demand is

$$
\Delta \log Y \approx \sum_{i} \lambda_{i} \Delta \log A_{i}+\sum_{f} \lambda_{f} \Delta \log L_{f}
$$

where $\lambda_{i}$ is the sales of producer $i$ as a share of GDP and $\lambda_{f}$ is the income of factor $f$ as a

\footnotetext{
${ }^{1}$ Although we take the shocks to be exogenous, we recognize that the shocks themselves can be endogenous responses in a larger model. For instance, labor supplied may be falling due to nominal rigidities or productivity may be falling due to firm exits. In fact, in a companion paper, Baqaee and Farhi (2020), we study these endogenous mechanisms. However, conditional on the quantity of each factor supplied, the productivity of each producer, and the composition of final demand, aggregate output is pinned down, and we study the nonlinearities in the resulting mapping from shocks to aggregate output.
} 
share of GDP. In other words, to a log-linearized first-order approximation, the change in real GDP is the sales-share weighted sum of supply shocks. This macro envelope theorem has surprising implications. It says that to a first-order, changes in the composition of demand have no effect on real GDP. It also says that to a first-order, the disaggregated structure of the economy matters only through the initial sales and factor shares.

These serendipities disappear when we go beyond the first order to capture nonlinearities. Building on some earlier work in Baqaee and Farhi (2019b), we generalize this aggregation equation to higher orders. We show that nonlinearities depend on equilibrium changes in sales and factor shares in response to the shocks. We also characterize the equilibrium changes in sales and factor shares by means of propagation equations. These propagation equations depend on all the shocks, including the shocks to the composition of final demand. ${ }^{2}$ They also depend on the disaggregated structure of the economy such as the elasticities of substitution in production and in consumption and the input-output matrix.

We emphasize two key implications. First, nonlinearities amplify negative supply shocks if these shocks are heterogenous and if sectors are complements throughout the input-output network, since then the worst-affected sectors become supply bottlenecks that drag the rest down with them. These complementarities are more important for transitory shocks such as the pandemic, as firms and households find it harder to substitute their production and consumption plans on short notice.

Second, nonlinearities amplify negative supply shocks if these shocks come together with shocks to the composition of final demand that shift final demand towards the worstaffected sectors, directly and indirectly through their supply chains. Changes in final demand are likely to be important during the pandemic. For instance, in the US, just as household preferences shifted towards consumption of more meat from grocery stores, some major meat-packing plants closed due to the pandemic. Or in France, just as households switched to more online shopping, Amazon was ordered to shutdown many of its warehouses to protect its workers. Of course, sometimes, changes in final demand could also mitigate the macro impact of the negative supply shocks, for example, when households' preferences move away from restaurants at the same time as the supply of restaurant meals goes down.

\footnotetext{
${ }^{2}$ Stability of final demand or preferences is an almost universal assumption in macroeconomics, particularly in the theory of aggregation. However, the Covid-19 crisis very likely changed household preferences, and so modelling these changes in final demand is essential. We define and characterize both real GDP and changes in welfare in this context. When final demand is unstable, real GDP acquires some very exotic (path-dependent) properties. One of the novel theoretical contributions of this paper is to tackle the problem of unstable final demand.
} 
We quantify the importance of nonlinearities using disaggregated data from the U.S. The shocks are large and heterogeneough enough that nonlinearities can amplify the impact of the shocks by $10 \%-100 \%$ depending on the horizon of analysis and the size of the underlying shocks.

We also extend our baseline analysis by studying cases where factor supplies are endogenous. In particular, we analyze the value of reallocating factors across the different sectors. Reallocations can tame nonlinearities and mitigate the negative impact of the shocks by reinforcing weak links. ${ }^{3}$ We also characterize the response of aggregate output in situations where the supply of labor and amount of capital utilization vary endogenously in response to changes in factor rewards. These extensions are important to assess the tradeoffs in the policy response to the crisis, such as the extension of unemployment insurance or the provision of business loans contingent on the preservation of payrolls.

The outline of the paper is as follows. In Section 2, we define the environment and set up the model. In Section 3, we state the basic comparative statics (for GDP and disaggregated sales and prices), and work through some illustrative examples. In Section 4, we extend the basic model to allow for endogenous factor supply and factor reallocation. In Section 6, we perform some preliminary quantitative exercises to gauge the importance of nonlinearities. We conclude in Section 7.

\section{Related Literature}

First, and foremost, the paper is part of the literature on economic effects of the Covid19 crisis. In particular, this paper is related to Barrot et al. (2020) who study the effect of Covid-19 using a quantitative production network. Our approach differs from theirs since we focus on analytical characterizations and a different set of thought experiments. This paper is also related Bodenstein et al. (2020), who analyze optimal shutdown policies in a two-sector model. Once again, our approach differs since we focus on positive rather than normative questions. Bonadio et al. (2020) analyze the effects of Covid-19 in an open-economy context.

Other economics papers studying the effects of Covid-19 include Eichenbaum et al. (2020a,b), Dingel and Neiman (2020), Mongey et al. (2020), Berger et al. (2020), Alvarez et al. (2020), Atkeson (2020a,b), Bethune and Korinek (2020), Guerrieri et al. (2020), Bigio et al. (2020), Caballero and Simsek (2020), Faria-e Castro (2020), Gourinchas (2020), Jones et al. (2020), Jorda et al. (2020), Kaplan et al. (2020), Krueger et al. (2020), Jorda et al. (2020), Bodenstein et al. (2020), Barrot et al. (2020), Barro et al. (2020), Fernández-Villaverde and

\footnotetext{
${ }^{3}$ See e.g. Jones (2011) and Baqaee and Farhi (2019b).
} 
Jones (n.d.), Hall et al. (2020), Glover et al. (2020), Fornaro and Wolf (2020), Acemoglu et al. (2020), Cakmakli et al. (2020), and Gourinchas et al. (2020).

This paper is also related to previous work by the authors. In particular, the paper builds on the analysis of nonlinearities in production networks in Baqaee and Farhi (2019b). We extend the analysis in that paper by studying real GDP and welfare in environments with unstable final demand, provide additional analytical characterizations, and extend that analysis to cover endogenous factor supply and reallocation. Of course, our quantitative application to the Covid-19 crisis is also new. Furthermore, this paper is related to a companion paper, Baqaee and Farhi (2020), which studies how nominal rigidities can provide an endogenous mechanism for the reductions in factor supply that are taken as given in this paper.

Of course, our analysis is also related to many non-Covid related papers, in particular, the literature on production networks which are summarized in Baqaee and Farhi (2019b) or Carvalho and Tahbaz-Salehi (2019). In future versions of this paper, we hope to expand our discussion of this and other related literatures.

\section{Setup}

In this section, we set up the basic model, define the shocks, the outcomes of interest, and the relevant notation. This section also contains a detailed discussion of how we define real GDP and welfare.

\subsection{Environment and Equilibrium}

The model has a set of producers $\mathcal{N}$, and a set of factors $\mathcal{G}$ with supply functions $L_{f}$. We write $\mathcal{N}+\mathcal{G}$ for the union of these two sets. With some abuse of notation, we also denote by $\mathcal{N}$ and $\mathcal{G}$ the cardinalities of these sets. The output of each producer is produced using intermediate inputs and factors, and is sold as an intermediate good to other producers and as a final good.

Final demand. Final uses $c_{i}$ of the different goods $i$ arise from the maximization of a constant-returns-to-scale final-demand aggregator

$$
\max _{\left\{c_{i}\right\}} \mathcal{D}\left(c_{1}, \ldots, c_{\mathcal{N}} ; \omega_{\mathcal{D}}\right)
$$


subject to the final-demand budget constraint

$$
\sum_{i \in \mathcal{N}} p_{i} c_{i}=\sum_{f \in \mathcal{G}} p_{f} L_{f}+\sum_{i \in \mathcal{N}} \pi_{i}
$$

where $p_{i}$ and $c_{i}$ are the price and final quantity of good $i, p_{f}$ is the wage of factor $f, \pi_{i}$ is the profits of the producer of good $i$. Ordinarily, it is taken for granted that $\mathcal{D}\left(\boldsymbol{c} ; \omega_{\mathcal{D}}\right)$ must equal real GDP. However, and crucially for our application, we allow for a demand shifter $\omega_{\mathcal{D}}$ which can change the shape of the final demand function. This can represent how household preferences over different consumption baskets change. For instance, if households prefer more groceries and less cruises as a result of the epidemic. If $\omega_{\mathcal{D}}$ changes, then as we discuss, real GDP no longer measures changes in the final demand index.

Producers. The output $y_{i}$ of good $i$ arises from the maximization of profits

$$
\pi_{i}=\max _{\left\{y_{i}\right\},\left\{x_{i j}\right\},\left\{L_{i f}\right\}} p_{i} y_{i}-\sum_{j \in \mathcal{N}} p_{j} x_{i j}-\sum_{f \in \mathcal{G}} p_{f} L_{i f}
$$

subject to a technological constraint described by a constant-returns production function

$$
y_{i}=A_{i} F_{i}\left(x_{i 1}, \ldots, x_{i \mathcal{N}}, L_{i 1}, \ldots, L_{i \mathcal{G}}\right),
$$

where $A_{i}$ is a Hicks-neutral productivity shifter and $y_{i}$ is total output.

Equilibrium. Given factor supplies $L_{f}$, productivities $A_{i}$, and demand shifters $\omega_{\mathcal{D}}$, an equilibrium is a set of prices $p_{i}$, factor wages $p_{f}$, intermediate input choices $x_{i j}$, factor input choices $l_{i f}$, outputs $y_{i}$, and final demands $c_{i}$, such that: each producer maximizes its profits subject to its technological constraint; final uses maximizes the final-demand aggregator subject to the final-demand budget constraint; and the markets for all goods and factors clear.

\subsection{Discussion}

Shocks. We will focus on three types of shocks: shocks to factor supplies, shocks to productivities, and shocks to demand. We believe that they are all important in the current context. Shocks to factor supplies could result, for example, from labor market retrenchment to avoid contagion perhaps because of confinement. Shocks to productivities could 
result, for example, from the reorganization of production such as working from home, or from the necessity of repurposing and repackaging some inputs for direct consumption vs. in outside venues. Shocks to demand could result, for example, from changes in consumption patterns arising from the fear of contagion. It would perhaps be more appropriate to refer to them as shocks to the composition of demand for different goods, but we call them shocks to demand for brevity.

Both for shocks to factor supplies and for shocks to demand, we stress that these changes in behavior could be voluntary if the agents modify their actions by themselves (internal effects), or involuntary if the government induces them through policy to correct externalities (external effects). For positive questions, say about the behavior of real GDP, this distinction is largely irrelevant. However, it matters when we turn to normative questions in Section 5.

Variable returns to scale. Despite specifying constant-returns cost functions, our setup can accommodate decreasing returns to scale. This is because, as pointed out by McKenzie (1959), variable returns to scale can be modeled with constant returns to scale and producer-specific fixed factors.

Non-Hicks-neutral shocks. Although the model is written in terms of Hicks-neutral productivity shocks, this is done without loss of generality. We can always capture nonneutral (biased) productivity shocks to the use of input $j$ by producer $i$ by introducing a fictitious producer buying input $j$ and selling to producer $i$ with a linear technology, and by considering Hicks-neutral shocks to this fictitious producer. For example, this can be important in the context of a pandemic because changes in the organization of production due to confinement and protection measures are likely to affect the productivities of labor an capital differently.

Investment. Investment can be modeled in different ways. The first possibility is to put investments in final demand. We then apply the model within a period. Changes in investment demand arising from expectations about future periods and states are then captured by demand shocks. The second possibility is to think of investments as intermediate goods in a dynamic stochastic model. We then apply the model inter-temporally and across states by indexing commodities by periods and states, and by letting final demand represent consumption not only within periods but also across periods and states. See footnote 5 below for more details. 


\subsection{Input-Output Definitions}

We define input-output objects such as input-output matrices, Leontief inverse matrices, and Domar weights associated with a competitive equilibrium. To make the exposition more intuitive, we slightly abuse notation in the following way. For each factor $f$, we interchangeably use the notation $L_{i f}$ or $x_{i(\mathcal{N}+f)}$ to denote its use by producer $i$, and the notation $L_{f}$ or $y_{f}$ or to denote total factor supply. We define final demand as an additional good produced by producer 0 according to the final demand aggregator. We interchangeably use the notation $c_{i}$ or $x_{0 i}$ to denote the consumption of good $i$ in final demand. We write $1+\mathcal{N}$ for the union of the sets $\{0\}$ and $\mathcal{N}$, and $1+\mathcal{N}+\mathcal{G}$ for the union of the sets $\{0\}, \mathcal{N}$, and $\mathcal{G}$. In other words, we add final demand and the factors into the IO matrix.

Input-output matrix. We define the input-output matrix to be the $(1+\mathcal{N}+\mathcal{G}) \times(1+$ $\mathcal{N}+\mathcal{G}$ ) matrix $\Omega$ whose $i j$ th element is equal to $i$ 's expenditures on inputs from $j$ as a share of its total revenues

$$
\Omega_{i j} \equiv \frac{p_{j} x_{i j}}{p_{i} y_{i}}=\frac{p_{j} x_{i j}}{\sum_{k \in \mathcal{N}+\mathcal{G}} p_{k} x_{i k}} .
$$

The input-output matrix $\Omega$ records the direct exposures of one producer to another, forward from upstream to downstream in costs, and backward from downstream to upstream in demand.

Leontief inverse matrix. We define the Leontief inverse matrix as

$$
\Psi \equiv(I-\Omega)^{-1}=I+\Omega+\Omega^{2}+\ldots
$$

The Leontief inverse matrix $\Psi$ records instead the direct and indirect exposures through the supply chains in the production network, forward from upstream to downstream in costs, and backward from downstream to upstream in demand. This can be seen most clearly by noting that $\left(\Omega^{n}\right)_{i j}$ measures the weighted sums of all paths of length $n$ from producer $i$ to producer $j$. 
Nominal GDP and Domar weights. Nominal GDP or nominal output is the total sum of all final expenditures

$$
G D P=\sum_{i \in N} p_{i} c_{i}=\sum_{i \in N} p_{i} x_{0 i}
$$

We define the Domar weight $\lambda_{i}$ of producer $i$ to be its sales as a fraction of GDP

$$
\lambda_{i} \equiv \frac{p_{i} y_{i}}{G D P}
$$

Note that $\sum_{i \in N} \lambda_{i}>1$ in general since some sales are not final sales but intermediate sales. Note that the Domar weight $\lambda_{f}$ of factor $f$ is simply its total income share.

The accounting identity $p_{i} y_{i}=p_{i} x_{0 i}+\sum_{j \in \mathcal{N}} p_{i} x_{j i}=\Omega_{0 i} G D P+\sum_{j \in \mathcal{N}} \Omega_{j i} \lambda_{j} G D P$ links the Domar weights to the Leontief inverse via

$$
\lambda_{i}=\Psi_{0 i}=\sum_{j \in \mathcal{N}} \Omega_{0 j} \Psi_{j i}
$$

where $\Omega_{0 j}=\left(p_{j} x_{0 j}\right) /\left(\sum_{k \in \mathcal{N}+\mathcal{G}} p_{k} x_{0 k}\right)=\left(p_{j} c_{j}\right) / G D P$ is the share of good $j$ in final expenditure.

\subsection{Nested-CES Economies}

We call an economy nested CES if all the production functions of all the producers (including final demand) are of the nested-CES form with Hicks-neutral technical change at the level of each nest. Any nested-CES economy, with an arbitrary number of producers, factors, CES nests, elasticities, and intermediate input use, can be re-written in what we call standard form, which is more convenient to study. To write a nested-CES economy in standard form, we simply treat each CES aggregator as though it is a distinct producer. Since the boundaries of the firm are irrelevant, this simply amounts to a relabelling of the set of producers.

A CES economy in standard form is defined by a tuple $(\bar{\omega}, \theta, F)$. The $(1+\mathcal{N}+\mathcal{G}) \times$ $(1+\mathcal{N}+\mathcal{G})$ matrix $\bar{\omega}$ is a matrix of input-output parameters. The $(1+\mathcal{N}) \times 1$ vector $\theta$ is a vector of microeconomic elasticities of substitution. Each good $i \in \mathcal{N}$ is produced with the production function

$$
\frac{y_{i}}{\bar{y}_{i}}=\frac{A_{i}}{\bar{A}_{i}}\left(\sum_{j \in \mathcal{N}+\mathcal{G}} \bar{\omega}_{i j}\left(\frac{x_{i j}}{\bar{x}_{i j}}\right)^{\frac{\theta_{i}-1}{\theta_{i}}}\right)^{\frac{\theta_{i}}{\theta_{i}-1}}
$$


where $x_{i j}$ are intermediate inputs from $j$ used by $i$. We represent final demand as the purchase of good 0 from producer 0 producing the final good

$$
\frac{y_{0}}{\bar{y}_{0}}=\left(\sum_{j \in \mathcal{N}+\mathcal{G}} \bar{\omega}_{0 j} \frac{\omega_{0 j}}{\bar{\omega}_{0 j}}\left(\frac{x_{0 j}}{\bar{x}_{0 j}}\right)^{\frac{\theta_{0}-1}{\theta_{0}}}\right)^{\frac{\theta_{0}}{\theta_{0}-1}}
$$

where $\omega_{0 j}$ is a demand shifter. In these equations, variables with over-lines are normalizing constants equal to the values at some initial competitive equilibrium and we then have $\bar{\omega}=\bar{\Omega} .{ }^{4}$ To simplify the notation below, we think of $\omega_{0}$ as a $1 \times(1+\mathcal{N}+\mathcal{G})$ vector with $k$-th element $\omega_{0 k}$. Changes in $\omega_{0}$ are how we model changes in the composition of demand.

\subsection{Real GDP and Welfare}

A main contribution of this paper is to analyze how changes in the composition of demand affect real GDP (aggregate output) and welfare. Shocks to the composition of demand are typically ruled out in growth-accounting, since real GDP acquires undesirable properties when final demand is unstable. However, real GDP is what we measure, and so in this paper, we are interested in understanding its behavior, warts and all. We start by discussing how we define real GDP in disaggregated economies, why real GDP becomes an exotic object when there are demand shocks, and then we discuss how we measure welfare, and why it differs from real GDP.

Real GDP. In the next sections, we provide comparative statics describing how, starting from an initial competitive equilibrium, the competitive equilibrium changes in response to changes in factor supplies $L_{f}$, productivities $A_{k}$, and demand shifters $\omega_{0 k}$. We compute real GDP using the artificial data generated by the model by applying the idealized procedure used by national-income accountants with the actual data. Consider a path indexed by $s$ for the factor supplies $L_{f, s}$, productivities $A_{k, s}$, and demand shifters $\omega_{0 k, s}$. Index the corresponding competitive equilibrium by $s$. We provide first- and second-order approximations in $d s=s-\bar{s}$ around an initial equilibrium corresponding to $\bar{s}$. Equivalently, these can be thought of as first- and second-order approximations in the shocks to pro-

\footnotetext{
${ }^{4}$ Note that when mapping the original economy to the re-labeled economy, the different nests in final demand are mapped intro different producers $j$. To simplify the exposition, we have imposed that there are only demand shocks in the outermost nest mapped to producer 0 . It is easy to generalize the results to allow for demand shocks in all the nests corresponding to final demand.
} 
ductivities $\Delta \log A_{k}=\int d \log A_{s}$, factor supplies $\Delta \log L_{f, s}=\int d \log L_{f, s}$, and demand $\Delta \log \omega_{0 k}=\int d \log \omega_{0 k, s}$. We also provide some exact results.

Global changes in output or real GDP are defined along this path by integrating local changes in real GDP

$$
\Delta \log Y=\int d \log Y_{s}
$$

where local changes in real output are obtained by deflating changes in nominal GDP using changes in the GDP deflator

$$
d \log Y_{s}=d \log G D P_{s}-d \log P_{s}
$$

and where changes in GDP deflator are defined as the weighted average of changes in final-demand prices with weights given by final demand shares

$$
d \log P_{s}=\sum_{i \in N} \Omega_{0 i, s} d \log p_{i, s}
$$

Defined in this way, as long as there are no demand shocks along the path $\left(\omega_{0 k, s}=\right.$ $\bar{\omega}_{0 k, s}$ for all $k$ and $\left.s\right)$, local changes in real GDP $d \log Y_{s}$ coincide with local changes in the final-demand quantity index $d \log y_{0, s}$. In other words, as long as the final demand aggregator is not changing, changes in real GDP coincide with the total derivative of the function $y_{0}\left(\left\{L_{f, s}\right\},\left\{A_{k, s}\right\}\right)$, which defines the final-demand quantity index $y_{0}$ as a function of factor supplies $L_{f, s}$ and productivities $A_{k, s}$ :

$$
\begin{aligned}
d \log Y_{s}=d \log y_{0, s}=\sum_{f \in F} \frac{\partial \log y_{0}\left(\left\{L_{f, s}\right\},\left\{A_{k, s}\right\}\right)}{\partial \log L_{f}} d \log L_{f, s} & \\
& +\sum_{k \in N} \frac{\partial \log y_{0}\left(\left\{L_{f, s}\right\},\left\{A_{k, s}\right\}\right)}{\partial \log A_{k, s}} d \log A_{k, s} .
\end{aligned}
$$

As long as the shocks evolve continuously, the same goes for global changes $\Delta \log Y=$ $\Delta \log y_{0}$ and so global changes in real GDP along a path depend only on the initial and end points of the path. Basically, real GDP can be defined in levels as $Y_{s}=y_{0, s}=$ $y_{0}\left(\left\{L_{f, s}\right\},\left\{A_{k, s}\right\}\right)$ and measures some well-defined notion of welfare.

However, when there are demand shocks, these useful relationships no longer hold. In general, local changes in real GDP $d \log Y_{S}$ do not correspond anymore to changes in the final demand quantity index, are not the total derivative of any function. Changes in real GDP $\Delta \log Y$ must instead be interpreted as a path integral along a non-conservative vector field. This means that real GDP along a path will depend not just on the beginning 
and end points of the path but on the whole path itself. These complications, which are usually ruled out, are important for our application, where household preferences over consumption baskets have likely changed. Intuitively, the problem arises from the fact that demand shocks directly change behavior without directly affecting transacted prices.

Issues of path depedence disappear when we turn to normative questions, since welfare can always be represented using a utility function, and so changes in utility do not depend on the path between the two end points. The same applies to disaggregated variables such as sales and factor shares, prices, and quantities. However these subtleties cannot be avoided for the positive questions involving aggregate output.

Welfare. Although not subject to any path-dependence problem, measuring welfare with shocks to the composition of demand is not completely straightforward either. And the distinction between preference-driven and policy-induced demand shocks, which did not matter for real GDP, matters a lot for welfare. In Section 5, we give a full treatment of both cases. Here, we limit our discussion to the case of preference-driven shocks to the composition of demand, and try to give a sense of the sort of issues that come up.

For example, suppose that the Covid crisis causes households to alter their ranking of different consumption baskets. For example, the pandemic makes households prefer more toilet paper and less cruises.

Because of Covid, the economy moves from an initial allocation $(p, c)$ to a different allocation $\left(p^{\prime}, c^{\prime}\right)$, where $p$ is the vector of prices and $c$ is the vector of consumption goods. Using post-Covid preferences, let $y_{0}^{\prime}$ be the utility attained at allocation $c^{\prime}$ and $y_{0}$ the utility attained at the allocation $c$. Let $e\left(p, y_{0}\right)$ be the household's expenditure function, using the post-Covid preferences. We define the change in welfare to be

$$
\Delta \log W=\log \left(\frac{e\left(p^{\prime}, y_{0}^{\prime}\right)}{e\left(p^{\prime}, y_{0}\right)}\right)
$$

In other words, how much must the household's income change to compensate them for having to consume the initial allocation rather than the post-shock allocation.

Note that this definition of welfare does not answer the question "how much would the household be willing to pay to eliminate Covid?" Instead, it answers the question "how much would the household be willing to pay to consume the original allocation?" That is, we compare the post-shock and pre-shock allocation using the household's new preferences. We do not compare the initial allocation using the pre-shock preferences to the post-shock allocation using the post-shock preferences. Since households cannot make a choice between a world with Covid and a world without Covid, preferences about such a comparison are 
not revealed by any of their choices.

When there are only shocks to preferences but no shocks to productivities or factor supplies, the first welfare theorem necessitates that the household must prefer allocation $y_{0}^{\prime}$ to allocation $y_{0}$ and so $\Delta \log W>0$.

Since we assume that preferences are homothetic, we can write $\Delta \log W=\log y_{0}^{\prime}-$ $\log y_{0}=\Delta \log y_{0}$. This means we can measure the change in welfare by simply using the change in the quantity-index $\Delta \log y_{0}$.

\section{Comparative Statics}

In this section, we derive general-equilibrium comparative static results. Although we will be mostly focused on changes in aggregate output, we will also characterize changes in disaggregated sales an factor shares, prices, and quantities.

We proceed in three steps. First, in Section 3.1, we derive an aggregation equation which gives a second-order approximation of changes in output as a function of the exogenous shocks and first-order changes in equilibrium sales and factor shares. Second, in Section 3.2, we derive a first-order approximation of changes in sales and factor shares as a function of the exogenous shocks and of microeconomic primitives. Combining the two first steps gives a second-order approximation of changes in output as a function of the exogenous shocks and of microeconomic primitives. Third, in Section 3.3, we specialize the model to provide illustrative examples as well as exact (not approximative) results.

\subsection{Aggregation Equation}

We start by giving a second-order approximation of changes in output as a function of the exogenous shocks and of first-order endogenous equilibrium changes in sales and factor shares. Here and in the rest of the paper, unless there is a risk of confusion, we use the simpler notation $\Omega$ and $\Psi$ instead of $\bar{\Omega}$ and $\bar{\Psi}$ to refer to the input-output objects at the initial equilibrium. Similarly, we use the notation and $\lambda$ instead of $\bar{\lambda}$ to refer to the initial sales and factor shares at the initial equilibrium, and $\Delta \log \lambda$ to indicate their changes.

Proposition 1. Changes in output around an equilibrium can be approximated up to the second 
order by the following aggregation equation:

$$
\begin{aligned}
\Delta \log Y \approx \sum_{k \in \mathcal{N}} \lambda_{k} \Delta \log A_{k}+\sum_{f \in \mathcal{G}} & \lambda_{f} \Delta \log L_{f} \\
& +\frac{1}{2} \sum_{k \in \mathcal{N}} \lambda_{k} \Delta \log \lambda_{k} \Delta \log A_{k}+\frac{1}{2} \sum_{f \in \mathcal{G}} \lambda_{f} \Delta \log \lambda_{f} \Delta \log L_{f} .
\end{aligned}
$$

The proof can be found in Appendix A.1. The first two terms on the right-hand side are the first-order terms, and the last two terms are the second-order terms.

The first-order terms are given by Hulten's theorem, which can be thought of as a version of the envelope theorem for output: the first-order effect on output of a productivity or factor supply shock in general equilibrium is given by the sales share of the shocked entity. For example, the reduction in output resulting from a reduction in the supply of a factor is bigger if the shock is bigger of if the share of this factor is larger.

The second order terms can be found by integrating

$$
\Delta \log Y=\int d \log Y_{s}=\sum_{k \in \mathcal{N}} \int \lambda_{k, s} d \log A_{k, s}+\sum_{f \in \mathcal{G}} \int \Lambda_{f, s} d \log L_{f, s}
$$

and approximating this expression to the second order. The second- and higher-order effects are given by the interactions of the shocks with the changes in the sales shares that take place in general equilibrium because of the shocks. For example, the reduction in output from a reduction in the supply of a factor is bigger not only if the shock is bigger and if the share of this factor is larger as in the first-order approximation, but also if the share of this factor increases because of this shock and all the other shocks, and if the shock increases the shares of the other entities hit with big negative shocks. The secondand higher-order effects capture nonlinearities: they matter more for large shocks; and they matter more for the interaction of the shocks.

Demand shocks $\Delta \log \omega_{0 k}$ do not appear directly in the aggregation equation. In fact, they have no first-order effects on output, and they have no second- and higher-order effects on output if there are no shocks to productivities and factor supplies. However, they do matter at the second- and higher-order in the presence of shocks to productivities and factor supplies because they induce changes in sales and factor shares. For example, the reduction in output from the reduction in the supply of a factor is larger if demand shifts towards goods that use this factor more directly and indirectly through their supply chains.

Neither the structure of the production network nor the elasticities of substitution in 
production and in final demand appear directly int the aggregation equation. The shocks, sales and factor shares, and changes in the equilibrium sales and factor shares caused by the shocks are sufficient statistics. As we shall see in Section 3.2 below, the structure of the network and the elasticities matter because they jointly influence the changes in the equilibrium sales and factor shares $\Delta \log \lambda$. We demonstrate this dependence by deriving propagation equations characterizing changes in equilibrium sales and factor shares as a function of microeconomic primitives. Combining the aggregation and propagation equations gives a second-order approximation of output as a function of microeconomic primitives. $^{5}$

\subsection{Propagation Equations}

We now give a first-order approximation of the endogenous equilibrium changes in sales and factor shares as a function of microeconomic primitives via propagation equations. These equations demonstrate the importance of the network, of the elasticities, and of their interactions.

We make use of the following notation and definition. For a matrix $M$, we denote by $M_{(i)}$ its $i$-th row by $M^{(j)}$ its $j$-th column. Given two $K \times 1$ vectors $X$ and $Y$ and an $1 \times K$ vector of $Z$ with $Z_{i} \in[0,1]$ and $\sum_{l \in K} Z_{l}=1$, we define $\operatorname{Cov}_{Z}(X, Y)=\sum_{l \in K} Z_{j l} X_{l} Y_{l}-$ $\left(\sum_{l \in K} Z_{j l} X_{l}\right)\left(\sum_{l \in K} Z_{j l} Y_{l}\right)$. It is a covariance of the random variables $X$ and $Y$ using the elements $l$ as realizations of random events with probabilities $Z_{l}$. We will oftentimes use this operator with $Z$ given by the $j$-th row $\Omega^{(j)}$ of the input-output matrix for some $j \in 1+\mathcal{N}$, and in this context, we will call the mapping from $(X, Y)$ to $\operatorname{Cov}_{\Omega^{(j)}}(X, Y)$ as the input-output covariance operator.

Proposition 2. Changes in prices can be approximated to the first order by the following forward propagation equations:

$$
\Delta \log p_{i} \approx-\sum_{k \in \mathcal{N}} \Psi_{i k} \Delta \log A_{k}+\sum_{g \in \mathcal{G}} \Psi_{i g}\left(\Delta \log \lambda_{g}-\Delta \log L_{g}+\Delta \log Y\right) .
$$

\footnotetext{
${ }^{5}$ If one desires to treat investments as intermediate goods rather than putting them in final demand as explained in Section 2.2, we must slightly change our procedure. In the first step, we apply the model to an enriched set of commodities indexed by dates and states. We compute changes in shares, prices, and quantities in this extended model by applying the results of Section 3.2. In the second step we compute the change in real GDP in a given period. To do so, we can still apply Proposition 1 . We must use the within period shares (the shares divided by the sum of the shares of the factors corresponding to that period) which can easily be computed from the first step. We must also count as factors the intermediate goods produced in previous periods and used in production in this period, the changes in the quantity of which is easily computed from the first step.
} 
Changes in sales shares and factor shares can be approximated to the first order by the following backward propagation equations:

$$
\lambda_{i} \Delta \log \lambda_{i} \approx \theta_{0} \operatorname{Cov}_{\Omega^{(0)}}\left(\Delta \log \omega_{0}, \Psi_{(i)}\right)+\sum_{j \in 1+\mathcal{N}} \lambda_{j}\left(\theta_{j}-1\right) \operatorname{Cov}_{\Omega^{(j)}}\left(-\Delta \log p, \Psi_{(i)}\right) .
$$

Changes in quantities can be approximated at the first order by $\Delta \log y_{i} \approx \Delta \log \lambda_{i}-\Delta \log p_{i}+$ $\Delta \log Y$.

The forward propagation equations describes how changes in prices propagate downstream (forward) through costs. A negative productivity shock $\Delta \log A_{k}$ to a producer $k$ upstream from $i$ increases the price of $i$ in proportion to how much $i$ buys from $k$ directly and indirectly as measured by $\Psi_{i k}$. Similarly an increase $\Delta \log p_{g}=\Delta \log \lambda_{g}-\Delta \log L_{g}+$ $\Delta \log Y$ in the price of factor $g$ increases the price of $i$ in proportion to the direct and indirect exposure of $i$ to $g$.

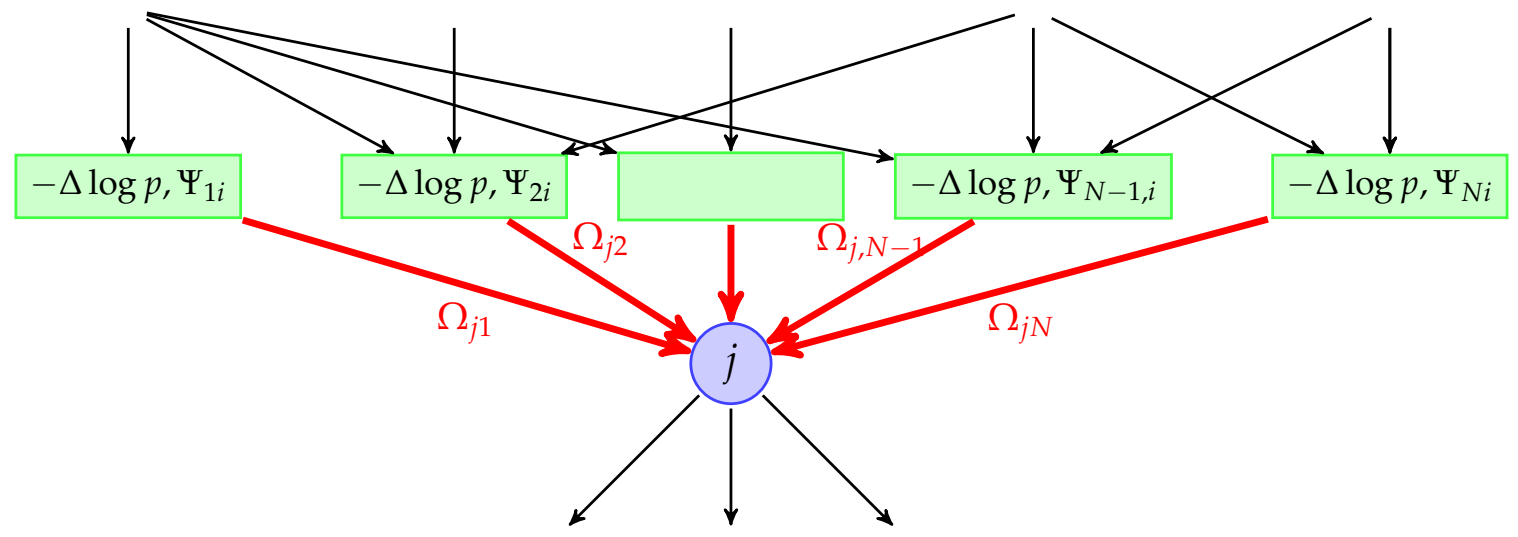

Figure 1: Graphical illustration of the IO covariance operator.

The backward propagations equations describe how changes in sales propagate upstream (backward) through demand. The first term $\sum_{k \in 1+N} \theta_{0} \operatorname{Cov}_{\Omega^{(0)}}\left(\Delta \log \omega_{0}, \Psi_{(i)}\right)$ on the right-hand side is the direct effect of demand shocks on the sales of $i$ in the absence of expenditure-switching due to substitution. The second term $\sum_{j \in 1+\mathcal{N}} \lambda_{j}\left(\theta_{j}-\right.$ 1) $\operatorname{Cov}_{\Omega^{(j)}}\left(-\Delta \log p, \Psi_{(i)}\right)$ on the right-hand side captures the changes in the sales of $i$ from substitutions by producers $j$ downstream from $i$. This is depicted in Figure 1. If producer $j$ has an elasticity of substitution $\theta_{j}$ below one so that its inputs are complements, then it shifts its expenditure towards those inputs $l$ with higher price increases $\Delta \log p_{l}$, and this increases the demand for $i$ if those goods $l$ buy a lot from $i$ directly and indirectly as measured by $\Psi_{l i}$. These expenditure-switching patterns are reversed when $\theta_{j}$ is above one so that the inputs of $j$ are substitutes, and when $\theta_{j}$ is equal to one they disappear. 
The following corollary combines the forward and backward propagation equations together to characterize changes in sales as a function of factor shares and factor shares as the solution of a system of $F$ linear forward-backward equations in $F$ unknowns.

Corollary 1. First-order changes in sales and factor shares solve the following linear system of of $1+\mathcal{N}+\mathcal{G}$ forward-backward equations in $1+\mathcal{N}+\mathcal{G}$ unknowns in the changes in the sales and factor shares $\Delta \log \lambda_{i}$ :

$$
\begin{aligned}
& \lambda_{i} \Delta \log \lambda_{i} \approx \theta_{0} \operatorname{Cov}_{\Omega^{(0)}}\left(\Delta \log \omega_{0}, \Psi_{(i)}\right) \\
& +\sum_{j \in 1+\mathcal{N}} \lambda_{j}\left(\theta_{j}-1\right) \operatorname{Cov}_{\Omega^{(j)}}\left(\sum_{k \in \mathcal{N}} \Psi_{(k)} \Delta \log A_{k}+\sum_{g \in \mathcal{G}} \Psi_{(g)}\left(\Delta \log \lambda_{g}-\Delta \log L_{g}\right), \Psi_{(i)}\right) .
\end{aligned}
$$

Specializing the system to factors $i=f \in \mathcal{G}$ yields a linear system of $\mathcal{G}$ equations in $\mathcal{G}$ unknowns in the changes factor shares $\Delta \log \lambda_{f}$ for $f \in \mathcal{G}$, with all the other sales shares explicitly solved for as a function of changes in factor shares.

Note that while changes in output $\Delta \log Y$ directly enter the forward propagation equations and hence indirectly the backward propagation equations in Proposition 2, they no longer enter the system of linear equations in sales and factor shares in Corollary 1. The reason is that changes in aggregate output wash out in the input-output covariances since they affect all prices in the same way. ${ }^{6}$

Our characterization is therefore block recursive. First, we solve a linear system of $\mathcal{G}$ forward-backward propagation equations in $\mathcal{G}$ unknowns in the changes in the factor shares $\Delta \log \lambda_{f}$ for $f \in \mathcal{G}$ from Corollary 1 . Then we get changes in the sales shares also from the forward-backward propagation equations in Corollary 1. Finally, we plug these changes in sales and factor shares in the aggregation equation in Proposition 2. Overall, our characterization is in closed form up to the solution of a linear system of $\mathcal{G}$ equations in $\mathcal{G}$ unknowns in the changes in the factor shares $\Delta \log \lambda_{f}$ for $f \in \mathcal{G}$.

\subsection{Illustrative Special Cases}

The aggregation and propagation equations described in Sections 3.1 and 3.2 give a secondorder approximation of the effects of shocks on output as a function of the shocks, the network, and the elasticities. This characterization is in closed form up to a system of linear equations in the changes in the factor shares. In this section, we specialize the model

\footnotetext{
${ }^{6}$ To see this, rewrite the forward propagation equations as $\Delta \log p_{i} \approx-\sum_{k \in \mathcal{N}} \Psi_{i k} \Delta \log A_{k}+$ $\sum_{g \in \mathcal{G}} \Psi_{i g}\left(\Delta \log \lambda_{g}-\Delta \log L_{g}\right)+\Delta \log Y$. This uses the fact that $\sum_{g \in \mathcal{G}} \Psi_{i g}=1$, which captures the fact that a uniform proportional increase in all factor prices results in a uniform proportional increase in all prices.
} 
in order to provide simpler versions of the second-order results and also exact (non-local) results.

We focus on the joint roles of the shocks, the network, and the elasticities in shaping nonlinearities. In Section 3.3.1, we focus on Cobb-Douglas economies and illustrate the role of the network with shocks to the composition of demand. In Section 3.3.2, we look at economies with uniform elasticities of substitution, which we show have a surprising network-irrelevance property, and we zoom in on the role of elasticities and shocks to factor supplies. In Section 3.3.3, we investigate a special class of networks where the joint roles of the elasticities, the input-output network, and the shocks can be handled in a simple way.

In each case, we provide general results and concrete applications in the context of Covid. In particular, in Section 3.3.2, we present a numerical illustration demonstrating the quantitative importance of nonlinearities.

\subsubsection{Cobb-Douglas}

We start with the Cobb-Douglas case where all the elasticities of substitution are equal to one.

Proposition 3. Suppose that all the elasticities of substitution are equal to one $\left(\theta_{j}=1\right.$ for all $j \in 1+\mathcal{N})$. Then changes in output can be approximated up to the second order by

$$
\begin{aligned}
\Delta \log Y \approx \sum_{k \in \mathcal{N}} \lambda_{k} \Delta \log A_{k} & +\sum_{f \in \mathcal{G}} \lambda_{f} \Delta \log L_{f} \\
& +\frac{1}{2} \operatorname{Cov}_{\Omega^{(0)}}\left(\Delta \log \omega_{0}, \sum_{k \in \mathcal{N}} \Psi_{(k)} \Delta \log A_{k}+\sum_{f \in \mathcal{G}} \Psi_{(f)} \Delta \log L_{f}\right) .
\end{aligned}
$$

Changes in sales and factor shares are given in Appendix A.3.

In the absence of shocks to demand, output is $\log$-linear in the shocks $\Delta \log Y=$ $\sum_{k \in \mathcal{N}} \lambda_{k} \Delta \log A_{k}+\sum_{f \in \mathcal{G}} \lambda_{f} \Delta \log L_{f}$. In this case, the first-order approximation provided by Hulten's theorem is exact. It is only when shocks to demand occur in conjunction with shocks to productivities or factor supplies that nonlinearities appear.

Implications. To unpack the nonlinearities, consider a univariate reduction $\Delta \log L_{f}<0$ in the supply of factor $f$ and a shock to demand $\Delta \log \omega_{0}$. If the demand shock redirects (directly and indirectly) expenditures towards this factor, it increases its share and amplifies the reduction in output. Conversely, if the demand shock redirects expenditures 
away from this factor, it reduces its share and mitigates the reduction in output. Figure 2 illustrates such a scenario. We plot the change in output in response to a negative productivity shock in a Cobb-Douglas model with two sectors (each with an initial spending share of 1/2). In the 'No demand shock' scenario, preferences are being held constant, whereas, in the other two scenarios, preferences change as the shock unfolds. By the end point, when production has fallen by about $20 \%$ in the negatively affected sector, demand for that sector's goods have increased or fallen by about $40 \%$.

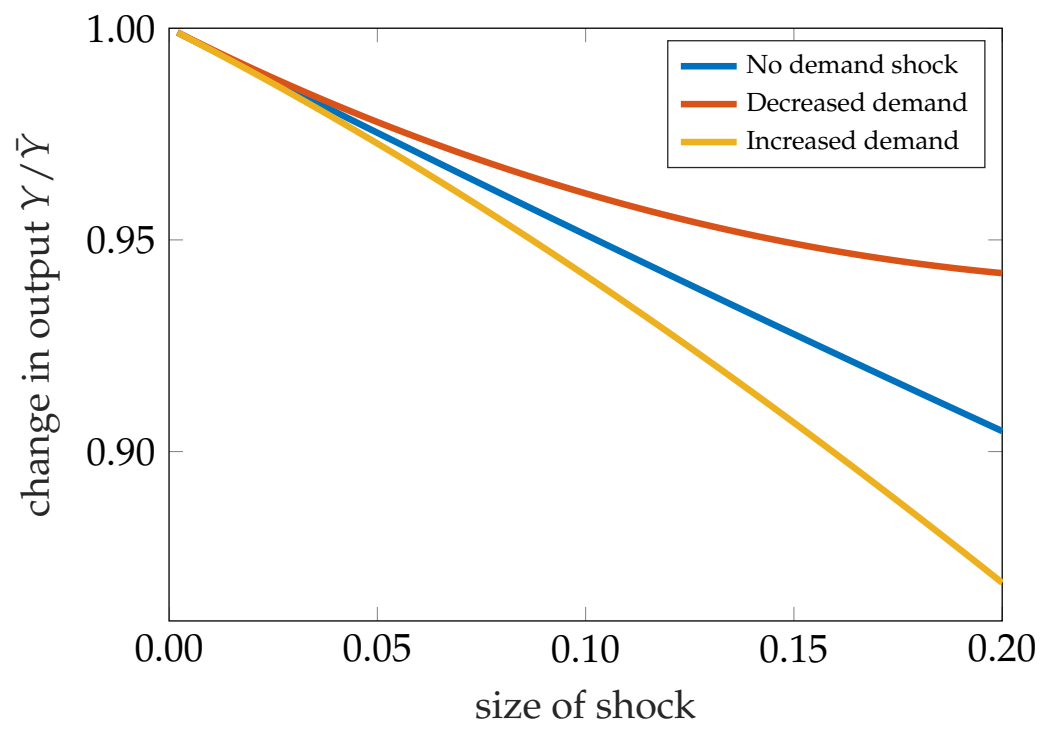

Figure 2: Output change $\Delta Y / \bar{Y}$ as a function of the size of a negative productivity shock to one sector, with or without an accompanying preference shock.

Concrete example. Concretely, consider Amazon's forced closure of its warehouses in France just as household preferences change in favor of demanding more goods from online retailers. Or meat packing plants in the US, like Smithfield (which accounts for $5 \%$ of US pork production) and JBS, closing due to an outbreak just as household preference shift in favor of consuming relatively more meat. In these cases, factors are being withdrawn concurrent with negative demand shocks for those factors. In these cases, nonlinearities amplify output losses because of the interaction of the two shocks .

Of course, this is not the only type of nonlinearity that matters. In the next example, we consider a different type of nonlinearity, one that does not rely on concurrent demand shocks. 


\subsubsection{Uniform Elasticities and Only Shocks to Factor Supplies}

To see the importance of nonlinearities in the absence of demand shocks, suppose that all the elasticities of substitution are the same and that there are are only shocks to factor supplies.

Proposition 4. Suppose that all the elasticities of substitution are the same $\left(\theta_{j}=\theta\right.$ for all $j \in$ $1+\mathcal{N}$ ) and that there are only shocks to factor supplies but no shocks to productivities and no shocks to demand. Then have an exact expression for changes in output:

$$
e^{\Delta \log Y}=\left(\sum_{f \in \mathcal{G}} \bar{\lambda}_{f}\left(e^{\Delta \log L_{f}}\right)^{\frac{\theta-1}{\theta}}\right)^{\frac{\theta}{\theta-1}} .
$$

We also have fully-solved-out approximations of changes in output to the second order:

$$
\Delta \log Y \approx \sum_{f \in \mathcal{G}} \lambda_{f} \Delta \log L_{f}+\frac{1}{2} \frac{\theta-1}{\theta} \operatorname{Var}_{\lambda}(\Delta \log L),
$$

where $\mathbb{E}_{\lambda}(\Delta \log L)=\sum_{f \in \mathcal{G}} \lambda_{f} \Delta \log L_{f}$ and $\operatorname{Var}_{\lambda}(\Delta \log L)=\sum_{f \in \mathcal{G}} \lambda_{f}\left(\Delta \log L_{f}-\mathbb{E}_{\lambda}(\Delta \log L)\right)$ are the expectation and variance of the shocks $\Delta \log L_{f}$ using the factor shares $\lambda_{f}$ as probabilities. Changes in sales and factor shares are given in Appendix A.3.

Proposition 4 shows that, in this special case, we can write the output function in closed-form, helping us to better see the nonlinearities.

Basically, under the assumptions of Proposition 4, output is given as a function of factor supplies by a simple CES aggregate production function with an elasticity of substitution $\theta$. This is because substitution patterns are uniform throughout the network between a given factor and all the other factors.

This surprising network-irrelevance result shows that in this case, the output effects of shocks to factor supplies are globally independent of the structure of the network. The network matters only through the initial factor shares $\bar{\lambda}_{f}$. In other words, even though there are many sectors and potentially complex and nonlinear supply chains, this information is entirely summarized by the initial factor income shares as long as the elasticities of substitution are uniform. Network irrelevance breaks if elasticities are not uniform, if there are shocks to productivities, or if there are shocks to demand.

Implications. In Proposition 3, we saw how demand shocks and supply shocks can interact to generate nonlinear losses even in a Cobb-Douglas model. However, without 
both types of shocks, a Cobb-Douglas model is loglinear. Once we deviate from CobbDouglas, output is nonlinear even when there are no demand shocks. Consider the case of complements $(\theta<1)$ with univariate shocks or multivariate shocks. All the patterns are reversed in the case of substitutes $(\theta>1)$. Overall, a lesson that emerges is that nonlinearities can amplify reductions in output when there are complementarities and when shocks are heterogeneous.

To see this, start with univariate shocks where there is only one factor $f$ with $\Delta \log L_{f} \neq$ 0 . Then nonlinearities amplify negative shocks and mitigate positive shocks compared to the first-order approximation. To see this, observe that the reduction in the supply of a factor causes its share to increase and hence amplifies the impact of the shock. The relative importance of these nonlinear effects and of the first-order effects increases with the size of the shock. This can be seen clearly using the approximations with $\operatorname{Var}_{\lambda}(\Delta \log L)=$ $\lambda_{f}\left(1-\lambda_{f}\right)\left(\Delta \log L_{f}\right)^{2}$. The second-order term is negative, and so changes in output are a concave function of the shock. These patterns are reversed for increases in the supply of a factor.

Consider next the case of complementarities and multivariate shocks where there are several factors $f$ with $\Delta \log L_{f} \neq 0$. The nonlinearities imply that the shocks interact. This can be seen clearly in the second-order terms where shocks enter through their variance $\operatorname{Var}_{\lambda}(\Delta \log L)$. In particular, when the shocks are homogenous so that $\Delta \log L_{f}=\Delta \log L_{g}$ for all $f$ and $g$, then factor shares do not change, and there are no nonlinearities. Relatedly, if all factors but $f$ experience the same negative shock, then the marginal impact of the same shock hitting factor $f$ is mitigated.

Numerical illustration. We only sketch the model and how it is calibrated. We refer the reader to Section 6 for more details on the calibration.

We consider a stylized version of the U.S. economy with 66 industries and industrial production functions with labor, capital, and intermediates. We focus on the short run and assume that labor and capital cannot be reallocated across industries. We assume uniform elasticities in production and in final demand equal to $\theta$.

The shock is a reduction in labor supplies calibrated to match the changes in hours worked by industry from the May 2020 BLS Economic News release. These numbers probably underestimate the actual reductions in hours because the underlying survey may not appropriately classify furloughed employees as temporarily unemployed. To allow for this possibility, we consider two scenarios: in the less extreme scenario, we assume that in each industry, the reduction in hours worked coincides with that given by the BLS; in the more extreme scenario, we inflate the log reduction in hours in each 
industry, reported by the BLS, by $50 \%$. The less extreme scenario reduces aggregate hours by around $13 \%$, whereas the more extreme scenario reduces aggregate hours by $20 \%$.

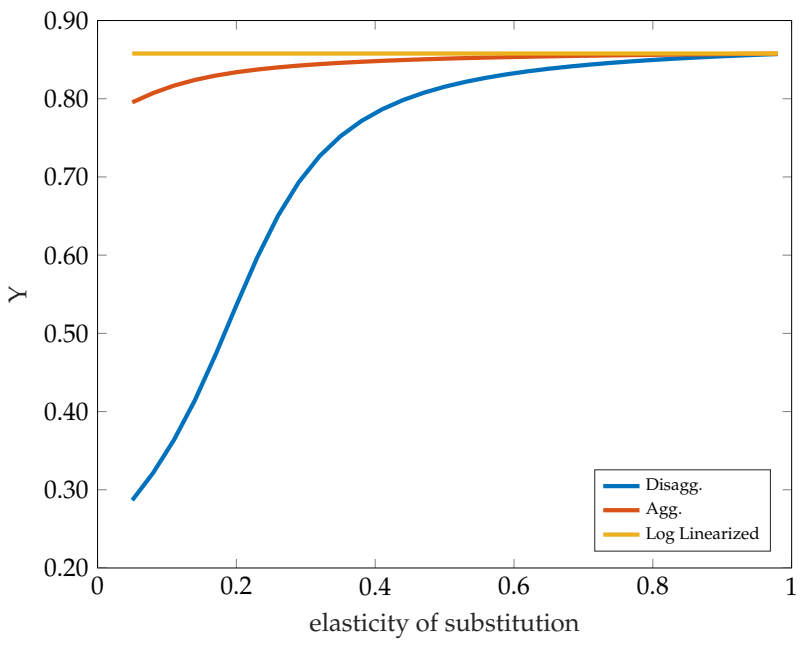

(a) more extreme scenario

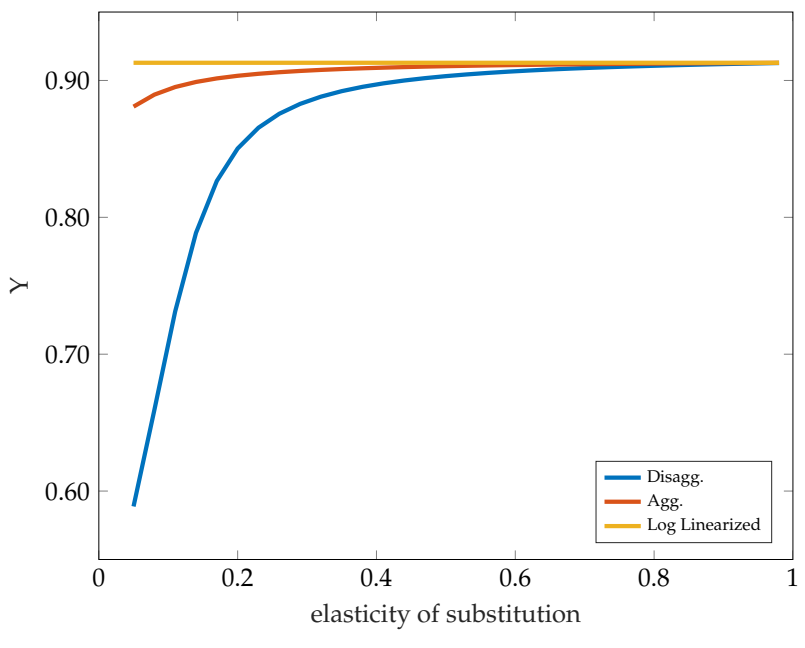

(b) less extreme scenario

Figure 3: Output relative to its initial value as a function of the (uniform) elasticity of substitution. In the less extreme scenario, the reduction in hours worked in each sector is equal to the number reported by the BLS; in the more extreme scenario, the reduction in hours worked in each sector is inflated by $50 \%$ compared to the number reported by the BLS.

We compare the outcome of this disaggregated model to that of an aggregate model where output is produced by an aggregate production function using a labor aggregate and a capital aggregate with the same elasticity of substitution $\theta$. The shares of the labor and capital aggregates are the total sales of all the labors and all the capitals in the disaggregated model. The labor and capital aggregates are log-linear Cobb-Douglas aggregates of the different labors and capitals with shares given by the relative shares of the different labors and the different capitals.

The two models produce the exact same output response in the Cobb-Douglas case when $\theta=1$, and both coincide with the first-order approximation given by Hulten's theorem. However, Figure 3 shows that as we lower $\theta$ below one to capture complementarities, output reductions in the disaggregated model are larger than in the aggregate model because the former is more nonlinear than the latter in both scenarios. This is because the shocks is very heterogeneous across sectors. If the shocks were homogeneous, then both models would generate the same output reductions.

The gap between the two models is sizable even for reasonable degrees of complementarity ( $\theta$ below 0.5 ) and becomes very large for high degrees of complementarity $(\theta$ close to zero). This is true for both scenarios but more so for the more extreme scenario. 
Which elasticity $\theta$ is empirically relevant is a tricky question, since aggregate output is not, in practice, a single CES aggregator. Nevertheless, our quantitative results in Section 6 suggest that in the short run and at our level of industrial aggregation, it is reasonable to assume a relatively low value of $\theta$, say 0.4 or lower. For example, with an elasticity of 0.4 : the reduction in output under the more extreme scenario is $14 \%$ in aggregate model and $20 \%$ in the disaggregated model (a $42 \%$ amplification); and the reduction in output under the less extreme scenario is $9 \%$ in the aggregate model and $11 \%$ in the disaggregated model (a $12 \%$ amplification).

Overall, this example demonstrates that because the shock is very heterogeneous, and because there are complementarities, it is important to use a disaggregated model to assess its impact on output. Although the two models coincide at the first order, nonlinearities amplify reductions in output significantly more in the disaggregated model than in the aggregate model that trivializes the heterogeneity in the shock.

Of course, this network-irrelevant case with uniform elasticities and no shocks to productivities or to the composition of demand is special. We already saw in the CobbDouglas case in Section 3.3.1 an example of how the network matters with shocks to the composition of demand. However, this example made it impossible to analyze how the different elasticities of substitution matter together with the network. The next example considers a class of networks where these interactions can be analyzed in a simple way.

\subsubsection{Acyclic Networks}

We now turn to the class of acyclic production networks, where the production network $\Omega$ is an acyclic graph. An example is depicted in Figure 4. These examples allow us to more easily see the importance of non-linearities in cases where the elasticities of substitution are not all the same.

The term acyclic here means that any two nodes are connected to one another by exactly one undirected path. In other words, each producer has a unique direct consumer. For a given producer $j \in 1+\mathcal{N}+\mathcal{G}$, we denote its unique immediately downstream consumers by $d(i)$ and the set of its immediately upstream suppliers by $u(i)$, with the convention that there is no-one downstream from final demand $d(0)=\varnothing$ and no-one upstream from factors $u(f)=\varnothing$ for $f \in \mathcal{G}$. We use the Kronecker symbol $\delta_{i j}=1$ if $i=j$ and $\delta_{i j}=0$ if $i \neq j .7$

\footnotetext{
${ }^{7}$ We can combine Propositions 4 and 5 to completely characterize a broader class of networks. Start with an acyclic production network. Each producer $j$ is a CES nest with an elasticity $\theta_{j}$. Using Proposition 4 , we can create a new network by replacing producer $j$ can be replaced by a subnetwork of producers buying from each other and from the inputs $i \in u(j)$ of producer $j$, and selling to each other and to the customer $d(j)$
} 


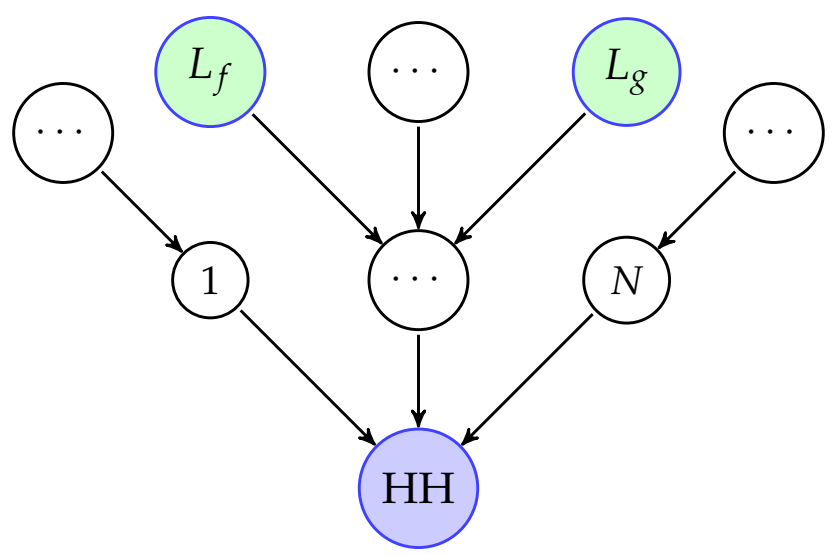

Figure 4: An acyclic economy, where the solid arrows represent the flow of goods. The factors are the green nodes. Each supplier (including factors) have at most one customer, whereas a single customer may have more than one supplier. Economies without cycles can be represented as directed trees with the household (final demand) denoted by $\mathrm{HH}$ being the root.

Proposition 5. Suppose that the production network is acyclic. Then we have exact an expression for changes in output recursively from upstream to downstream in the absence of shocks to demand:

$$
e^{\Delta \log Y}=e^{\Delta \log y_{0}}, \quad e^{\Delta \log y_{i}}=e^{\Delta \log A_{i}}\left(\sum_{j \in u(i)} \bar{\Omega}_{i j}\left(e^{\Delta \log y_{j}}\right)^{\frac{\theta_{i}-1}{\theta_{i}}}\right)^{\frac{\theta_{i}}{\theta_{i}-1}},
$$

where by definition $\Delta \log y_{i}=\Delta \log L_{f}$ if $i=f \in \mathcal{G}$ is a factor and where we define $\Delta \log A_{0}=0$. We also have fully-solved-out approximations of changes in output to the second order even with shocks to demand:

$$
\begin{aligned}
\Delta \log Y \approx & \sum_{k \in \mathcal{N}} \lambda_{k} \Delta \log A_{k}+\sum_{f \in \mathcal{G}} \lambda_{f} \Delta \log L_{f} \\
& +\sum_{j \in 1+\mathcal{N}} \frac{1}{2} \lambda_{j} \frac{\theta_{j}-1}{\theta_{j}} \operatorname{Var}_{\Omega^{(j)}}\left(\sum_{k \in \mathcal{N}} \Psi_{(k)} \Delta \log A_{k}+\sum_{f \in \mathcal{G}} \Psi_{(f)} \Delta \log L_{f}\right) \\
& \quad+\frac{1}{2} \theta_{0} \operatorname{Cov}_{\Omega^{(0)}}\left(\Delta \log \omega_{0}, \sum_{k \in \mathcal{N}} \Psi_{(k)} \Delta \log A_{k}+\sum_{f \in \mathcal{G}} \Psi_{(f)} \Delta \log L_{f}\right) .
\end{aligned}
$$

Changes in sales and factor shares are given in Appendix A.3.

of producer $j$. Final consumption in the sub-network corresponds to consumption by $d(j)$ in the original network, and factors in the sub-network correspond to the inputs $i \in u(j)$ producer $j$. The elasticities of substitution network are uniform and equal to $\theta_{j}$, and there are no shocks to productivities or to demand in the sub-network. The characterization given in Proposition 5 remains unchanged since the sub-network gives replicates the original CES production function of producer $j$. 
Such economies have a unique feasible allocation, because there is no option to allocate a given factor or good to different uses, and this is what makes them so tractable.

For our purposes, it is useful to think of such an economy as a probability tree. Each node is a history of events. The root of the tree is the is producer 0 corresponding to final demand. The leaves of the tree are the factors. Each node has a set of upstream branches and is attached to a unique downstream branch. The probability of node 0 is one, the conditional probability of reaching node $j \in u(i)$ in one more step conditional on being at node $i$ is $\Omega_{i j}$. More generally, the probability of reaching node $j$ in any number of steps conditional on being at node $i$ is $\Psi_{i j}$. The unconditional probability of reaching node $i$ is given by its sales share $\lambda_{i}$. We also think of the shocks to factor productivities $\Delta \log A_{k}$ and factor supplies $\Delta \log L_{f}$ as realizations of random variables $\Delta \log A$ and $\Delta \log L$. Finally, we think of demand shocks $\Delta \log \omega^{(0) \prime}$ as a change of probability measure.

The different terms in Proposition 5 receive a probabilistic interpretation. The term $\operatorname{Var}_{\Omega^{(j)}}\left(\sum_{k \in \mathcal{N}} \Psi_{(k)} \Delta \log A_{k}+\sum_{f \in \mathcal{G}} \Psi_{(f)} \Delta \log L_{f}\right)$ is simply the variance conditional on history $j$ of the different expectations of the random variable $\Delta \log A+\Delta \log L$ conditional on the continuing histories of $j$. Similarly, the term $\operatorname{Cov}_{\Omega^{(0)}}\left(\Delta \log \omega_{0}, \sum_{k \in \mathcal{N}} \Psi_{(k)} \Delta \log A_{k}+\right.$ $\left.\sum_{f \in \mathcal{G}} \Psi_{(f)} \Delta \log L_{f}\right)$ is the impact of the change in probability measure on the expectation of the random variable $\Delta \log A+\Delta \log L$.

Implications. When the elasticities are not uniform, the structure of the production network matters even when there are only shocks to factor supplies. In particular, for a given set of shocks, changes in output are decreasing in all the elasticities of substitution $\theta_{j}$ at the second order, and the associated nonlinearities contribute to reducing output with complementarities $\left(\theta_{j}<1\right)$ and to increasing output with substituabilities $\left(\theta_{j}>1\right)$. How much each elasticity $\theta_{j}$ matters depends on the shocks and on the structure of the networks (the probabilities) through $\operatorname{Var}_{\Omega^{(j)}}\left(\sum_{f \in \mathcal{G}} \Psi_{(f)} \Delta \log L_{f}\right)$ (the conditional variance of the conditional expectation of $\Delta \log L){ }^{8}$

When there are shocks to productivities $\Delta \log A$ or demand $\Delta \log \omega_{0}$, the structure of the production network matters even when the elasticities are uniform. For example, a univariate productivity shock $\Delta \log A_{i}$ to a producer $i$ downstream from $j$ generates less nonlinearities at the second order than a corresponding univariate shock $\Delta \log A_{j}=$ $\left(\lambda_{i} / \lambda_{j}\right) \Delta \log A_{i}$ to $j$ renormalized by the relative size $\lambda_{i} / \lambda_{j}$ of $i$ and $j$ so that the two shocks have the same first-order effects. In the same example, when the elasticities are

\footnotetext{
${ }^{8}$ When the elasticities are uniform and equal to $\theta$ and there are only shocks to factor supplies, the secondorder term is proportional to $\sum_{j \in 1+\mathcal{N}} \lambda_{j} \operatorname{Var}_{\Omega^{(j)}}\left(\sum_{f \in \mathcal{G}} \Psi_{(f)} \Delta \log L_{f}\right)$ which can be seen as a decomposition of the unconditional variance $\operatorname{Var}_{\lambda}(\Delta \log L)$ into conditional expectations of variances of conditional expectations.
} 
not uniform, changes in output are independent of elasticities upstream from $i$ at the second order because they do not change its sales share at the first order.

Concrete example. As a concrete example imagine a simple structure with three sectors: food, brick-and-mortar retail, and online retail. Imagine that all sectors produce using different labors and that workers cannot be reallocated across sectors. Brick-andmortar retail $(B M R)$ and online retail $(O R)$ enter in a retail $(R)$ nest 1 , and that the retail nest and food $(F)$ enter in the final demand nest $D$. Imagine that brick-and-mortar retail downsizes and fires some of its workers $\left(\Delta \log L_{L M B R}<0\right)$ in an effort to mitigate contagion risk in a pandemic. Recognizing that brick-and-mortar retail and online retail are more substitutable than retail and food $\left(\theta_{R}>\theta_{D}\right)$ rather than equally substitutable $\left(\theta_{R}=\theta_{D}\right)$ mitigates the impact of the shock on output by $(1 / 2)\left(1 / \theta_{D}-\right.$ $\left.1 / \theta_{R}\right)\left(\lambda^{B M R} / \lambda^{R}\right)\left(1-\lambda^{B M R} / \lambda^{R}\right)\left(\Delta \log L_{L M B R}\right)^{2}$.

\section{Endogenous Factor Supplies}

In this section, we extend the model to allow for endogenous factor supplies. We focus on two margins: factor reallocation and labor-leisure/factor utilization. In Appendix A.5, we show how these two margins can also be analyzed jointly in one single model by modeling factor supply à la Roy. In the main body, we focus on communicating the same insights using a simpler specification.

\subsection{Factor Reallocation}

We start by exploring the role of factor reallocation. We use a simple second-order envelope analysis which can be conducted in the baseline model by either allowing or not some factors to be reallocated. In Appendix A.5, we show how to generalize these insights to endogenously smoother reallocations guided by finite elasticities of substitution in factor supplies by extending the baseline model by adding an endogenous Roy factor supply block.

Take two factors $h$ and $h^{\prime}$. Imagine that they are in fact the same underlying effective factor. By this we mean that factor $h^{\prime}$ could be used as a perfect substitute for factor $h$ and vice versa. Labor could therefore be reallocated not only between the different uses of $h$ and between the different uses of $h^{\prime}$ separately as in the baseline model, but also between all the different uses of $h$ and $h^{\prime}$ jointly. In addition, imagine that at initial equilibrium there would be no gain from these further reallocations between $h$ and $h^{\prime}$, which simply 
means that effective units of labor are getting the same wage if they are used as $h$ or as $h^{\prime}$. Without loss of generality, normalize the conversion of effective units between the two factors as one for one. Now imagine that there is a shock to demand, productivities, and supplies of factors.

We consider two thought experiments which capture different forms of reallocations. We refer to them as "sharing factor suppression" and "redeploying suppressed factors". Both illustrate the forces shaping the benefits of allowing factor reallocations, and conversely, the cost of preventing them. One of the reasons this is important is because many policies adopted to mitigate transitory shocks have the side effect of discouraging reallocations. For example, in a pandemic, one might be tempted to preserve organizational capital by keeping workers and capital attached to their initial employers for the duration of the shock. Their benefits notwithstanding, such policies also have costs and we study these costs.

\subsubsection{Sharing Factor Suppression}

In the first thought experiment, we assume that in the reallocation economy, the overall reduction in factor supply can be shared across $h$ and $h^{\prime}$ so that $\Delta \tilde{L}_{h}+\Delta \tilde{L}_{h^{\prime}}=\Delta L_{h}+\Delta L_{h^{\prime}}$. For example, this could be because the government seeks to remove a certain number of workers from the workforce in order to avoid the contagion of a virus. We then compare the response of this reallocation economy (denoted with hats) to that of the noreallocation economy up to the second order:

$$
\Delta \log \hat{Y}-\Delta \log Y \approx \frac{1}{2} \frac{1}{\frac{1}{\lambda_{h}}+\frac{1}{\lambda_{h^{\prime}}} \frac{\left(\Delta \log \lambda_{h}-\Delta \log \lambda_{h^{\prime}}\right)^{2}}{\lambda_{h^{\prime}}+\lambda_{h^{\prime}}}, \frac{1}{\sigma_{h^{\prime} h}}+\frac{\lambda_{h}}{\lambda_{h}+\lambda_{h^{\prime}}} \frac{1}{\sigma_{h h^{\prime}}}},
$$

where the expressions for the changes in factor shares $\Delta \log \lambda_{h}$ and $\Delta \log \lambda_{h^{\prime}}$ in the noreallocation economy are given functions of microeconomic primitives by Corollary 1. Here $\sigma_{h h^{\prime}}$ and $\sigma_{h^{\prime} h}$ are the (Morishima) macroeconomic elasticities of substitution between $h$ and $h^{\prime}$ taken as separate factors in the no-reallocation economy. ${ }^{9}$

Compared to the economy where $h$ and $h^{\prime}$ are stuck, the economy with reallocation removes the misallocation between $h$ and $h^{\prime}$. This misallocation is given by a Harberger triangle: it is proportional to the harmonic average of the elasticities of substitution between $h$ and $h^{\prime}$, and to the square of the implicit distortion wedges between $h$ and $h^{\prime}$ given by $\Delta \log \lambda_{h}-\Delta \log \lambda_{h^{\prime}}$. Removing this misallocation increases output by the size of this

\footnotetext{
${ }^{9}$ For example $\sigma_{h h^{\prime}}$ is defined as $1-1 / \sigma_{h h^{\prime}}=\left(d \log \lambda_{h^{\prime}}\right) /\left(d \log L_{h^{\prime}}\right)-\left(d \log \lambda_{h}\right) /\left(d \log L_{h^{\prime}}\right)$ and can be computed as a function of microeconomic primitves using Corollary 1.
} 
triangle. Allowing these sorts of reallocations can therefore mitigate the negative impact of the shocks on output.

\subsubsection{Redeploying Suppressed Factors}

In the second thought experiment, we assume that $\Delta \log L_{h}<0$ but that $\Delta \log L_{h^{\prime}}=0$. We now interpret $\Delta \log L_{h}<0$ as a binding reduction on how much factor $h$ can be used. In the reallocation economy, the released supply of factor $h$ can be reallocated to the uses of factor $h^{\prime}$ so that $\Delta \tilde{L}_{h}=\Delta L_{h}$ and $\Delta \tilde{L}_{h^{\prime}}=-\Delta L_{h}$. For example, this could be because the government seeks to remove a certain number of workers from specific occupations which are more prone to generating the contagion of a virus. We then compare the response of this reallocation economy (denoted with tildes) to that of the no-reallocation economy up to the second order by applying the results of Proposition 1 and Corollary 1 to the no-reallocation economy and to the reallocation economy.

In the sharing-factor-suppression experiment, the gains from allowing reallocations were only second order. By contrast, in this redeploying-suppressed-factors experiment, there are also first-order gains $-\lambda_{h} \Delta \log L_{h}$. Allowing these second sorts of reallocations is therefore even more important than removing the first sorts.

\subsubsection{Illustrative Examples}

Sharing factor suppression. As an example of the sharing-factor-suppression experiment, imagine that all elasticities of substitution are the same and equal to $\theta$, and that there are no shock to productivities and no shocks to demand. Then $\sigma_{h h^{\prime}}=\sigma_{h^{\prime} h}=\theta$ and $\Delta \log \lambda_{h}-\Delta \log \lambda_{h^{\prime}}=((\theta-1) / \theta)\left(d \log L_{h}-d \log L_{h^{\prime}}\right)$. The gains from reallocations are then given up to the second order by

$$
\Delta \log \hat{Y}-\Delta \log Y \approx \frac{1}{2} \frac{1}{\frac{1}{\lambda_{h}}+\frac{1}{\lambda_{h^{\prime}}}} \frac{(\theta-1)^{2}}{\theta}\left(\Delta \log L_{h}-\Delta \log L_{h^{\prime}}\right) .
$$

Reallocations spread the reductions in factor supply over $h$ and $h^{\prime}$ and this mitigates the resulting output losses by taming nonlinearities at the second order. These nonlinearities scale in $(\theta-1)^{2} / \theta$ and are therefore large when $\theta$ is far from one, either with strong complementarities or with strong substituabilities.

Redeploying suppressed factors. As an example of the redeploying-suppressed-factors experiment, continue to assume that all elasticities of substitution are the same and equal to $\theta$, and that there are no shock to productivities and no shocks to demand. Assume 
in addition that there are no shocks to the factors $f$ other than $h$ and $h^{\prime}$, so that the only shock is to the supply of factor $h$ (see Appendix A.4 for the general case). The gains from reallocations are then given up to the second order by

$$
\Delta \log \tilde{Y}-\Delta \log Y \approx \lambda_{h^{\prime}} \Delta \log \tilde{L}_{h^{\prime}}+\frac{\theta-1}{\theta} \lambda_{h}\left(1+\frac{\lambda_{h}}{\lambda_{h^{\prime}}}\right) \sum_{j \in 1+\mathcal{N}} \frac{1}{2}\left(\Delta \log L_{h}\right)^{2},
$$

where $\lambda_{h^{\prime}} \Delta \log \tilde{L}_{h^{\prime}}=-\lambda_{h} \Delta \log L_{h}+(1 / 2) \lambda_{h}\left(1-\lambda_{h} / \lambda_{h^{\prime}}\right)\left(\Delta \log L_{h}\right)^{2}$. There are two terms on the right-hand side of this expression which correspond to the first- and second-order effects of reallocations. Reallocations are always beneficial at the first order, but nonlinearities mitigate these benefits at the second order when there are complementarities $(\theta<1)$ because they increase the share of factor $h$ which experiences a reduction in supply and decrease that of factor $h^{\prime}$ which experiences an increase in supply. The results are reversed when there are substituabilities $(\theta>1)$ so that nonlinearities amplify the firstorder benefits of reallocations at the second order. These nonlinearities scale in $(\theta-1) / \theta$ and are therefore potentially much stronger for complementarities than for substituabilities. They also scale with $\left(\lambda_{h}+\lambda_{h^{\prime}}\right) / \lambda_{h^{\prime}}$ and are therefore stronger for a given $\lambda_{h}$ if the relative number of workers in $h^{\prime}$ vs. $h$ is smaller.

To shed light on the role of heterogeneous elasticities consider are no shocks to productivities, no shocks to demand, and no shocks to the factors $f$ other than $h$ and $h^{\prime}$, so that the only shock is to the supply of factor $h$ (see Appendix A.4 for the general case). The gains from reallocations are then given up to the second order by

$\Delta \log \tilde{Y}-\Delta \log Y \approx \lambda_{h^{\prime}} \Delta \log \tilde{L}_{h^{\prime}}+\sum_{j \in 1+\mathcal{N}} \frac{1}{2} \lambda_{j} \frac{\theta_{j}-1}{\theta_{j}}\left(\Psi_{\left(j h^{\prime}\right)}\left(\frac{\lambda_{h}}{\lambda_{h^{\prime}}}\right)^{2}+\left(\Psi_{(j h)}\right)^{2}\right)\left(\Delta \log L_{h}\right)^{2}$.

We focus on the nonlinearities captured by the second term on the right-hand side. These gains are larger if there are many large nests $j$ (as captured by $\lambda_{j}$ ) that substitute a lot across their inputs (as captured by $\theta_{j}>1$ ) and are directly and indirectly exposed to $h$ and $h^{\prime}$ (as captured by $\Psi_{\left(j h^{\prime}\right)}\left(\lambda_{h} / \lambda_{h^{\prime}}\right)^{2}+\Psi_{(j h)}^{2}$ ). Conversely, the gains are smaller if there are large nests $j$ that substitute little across their inputs (as captured by $\theta_{j}<1$ ) and are directly and indirectly exposed to $h$ and $h^{\prime}$.

As a concrete example imagine a simple structure with three sectors: restaurants, home deliveries of prepared meals, and online retail. To capture frictions to reallocation, imagine that all sectors produce using different labors, so that workers, who we assume would be equally productive in all sectors, cannot be reallocated across sectors in the noreallocation economy. Restaurants and home deliveries of prepared meals are substitutes 
in a food nest, and the food nest and online retail are complements in the final demand nest. Imagine that restaurants downsize their staffs in an effort to mitigate contagion risk in a pandemic. Restaurant workers might be reallocated to home deliveries of prepared meals, or to the online retail sector (a likely complement to restaurants). The benefits of a given amount of reallocation is then larger if it is of former rather than the latter kind.

\subsection{Labor-Leisure/Factor Utilization}

We now explore the role of endogenous factor supplies by introducing labor-leisure/ factor-utilization margins. Endogenous factor utilization/supply allows us to think about the fact that shocks may change factor rewards, and as factor rewards change, the quantity of factors supplied can change, further amplifying (or mitigating) the original shock.

\subsubsection{Extending the Analysis to Endogenous Factor Supplies}

Suppose factor supply curves have the form

$$
\Delta \log L_{f}=\Delta \log \mathcal{L}_{f}^{\text {price }}+\phi_{f} \Delta \log \left(p_{f} / p_{0}\right)-\gamma_{f} \Delta \log y_{0}
$$

where $\Delta \log \left(p_{f} / p_{0}\right)$ is the change in the real price of factor $f, \Delta \log y_{0}$ is the change in the quantity of the final good, and $\Delta \log \mathcal{L}_{f}^{\text {price }}$ is factor supply shock which could be preference-driven or government-induced. Note that $\Delta \log y_{0}$ coincides with changes in output $\Delta \log Y$ in the absence of shocks to demand, and with changes in welfare $\Delta \log W$ with preference-driven shocks to demand. There are different possible micro-foundations of such an equation for both labor and capital.

When $f$ is a form of labor, then the most obvious microfoundation is a standard laborleisure problem in utility. The case where $\phi_{f}=\gamma_{f}$ corresponds to the labor supply curve that can be derived from balanced-growth preferences. ${ }^{10}$

\footnotetext{
${ }^{10}$ Other microfoundations are also possible, for example, we could assume that it is supplied by a continuum of agents with balanced growth preferences who can each supply one or zero unit of labor. If they don't supply labor, they experience more leisure and collect some benefits $\bar{b}_{f}=\left(b_{f} / \bar{b}_{f}\right)\left(y_{0} / \bar{y}_{0}\right)^{1+\gamma_{f} / \phi_{f}}$ from the government, where $1+\gamma_{f} / \phi_{f}$ is the degree of indexation of benefits to final output. These benefits are financed by either by a uniform linear tax on factor income and benefits or by taxes on inelastic factors. Natural cases to consider are then $\gamma_{f}=-\phi_{f}$ (no indexation) and $\gamma_{f}=0$ (full indexation). If the utilities from leisure are Pareto distributed with parameter $\phi_{f}$ in the population, then get exactly the factor supply curve above with $\Delta \log \mathcal{L}_{f}=-\phi_{f} \Delta \log b_{f}$. If there is a uniform increase in the preference for leisure by $\Delta \log v_{f}$, perhaps because this allows to avoid getting infected by a virus, then we get $\Delta \log \mathcal{L}_{f}^{\text {price }}=-\phi_{f}\left(\Delta \log b_{f}+\Delta \log v_{f}\right)$. And if in addition the government deters work by these agents with an implicit tax $\Delta \log \tau_{f}$, then we get $\Delta \log \mathcal{L}_{f}^{\text {price }}=-\phi_{f}\left(\Delta \log b_{f}+\Delta \log v_{f}+\Delta \log \tau_{f}\right)$.
} 
We can also use this relationship to model endogenous capital utilization, similar to Christiano et al. (2005), where $\phi_{f}$ captures the convexity of the capital utilization cost. Under this formulation of capital utilization, we would have $\gamma_{f}=0$.

The factor supply equation can also be rewritten as

$$
\Delta \log L_{f}=\Delta \log \mathcal{L}_{f}^{\text {share }}+\frac{\phi_{f}}{1+\phi_{f}} \Delta \log \lambda_{f}+\frac{\phi_{f}-\gamma_{f}}{1+\phi_{f}} \Delta \log y_{0}
$$

where $\Delta \log \mathcal{L}_{f}^{\text {share }}=\left(1 /\left(1+\phi_{f}\right)\right) \Delta \log \mathcal{L}_{f}^{\text {price }}$ is the factor supply shock for a given factor share and utility, which already takes into account the mitigation of the response of the supply of the factor when its share rather than its price is held constant. Changes in the supply of the factor $\Delta \log L_{f}$ differs from $\Delta \log \mathcal{L}_{f}^{\text {share }}$ because of endogenous changes in factor supplies due to equilibrium changes in shares.

We can combine these equations with the aggregation equation in Proposition 1 and the forward-backward propagations equations in Corollary 1 to characterize the effects of shocks on output,ales, factor shares, prices, and quantities to the first order. Note that we do not obtain a general second-order characterization of changes in output because we only have first-order approximation of changes in factor shares and hence of changes in factor supplies.

\subsubsection{Illustrative Examples}

In this section, we give some illustrative examples.

Cobb-Douglas. We start with the case where all the elasticities of substitution in production and in final demand are equal to one $\left(\theta_{j}=1\right.$ for all $\left.j \in 1+\mathcal{N}\right)$. This case is tractable because changes in shares are given by a simple exact log-linear closed form. For simplicity, we also assume that $\phi_{f}=\gamma_{f}$ for all $f \in \mathcal{G}$. To a second order, changes in output are given by

$$
\begin{gathered}
\Delta \log Y \approx \sum_{k \in \mathcal{N}} \lambda_{k} \Delta \log A_{k}+\sum_{f \in \mathcal{G}} \lambda_{f} \Delta \log \mathcal{L}_{f}^{\text {share }}+\operatorname{Cov}_{\lambda}\left(\frac{\phi}{1+\phi}, \Delta \log \lambda\right) \\
+\frac{1}{2} \operatorname{Cov}_{\Omega}^{(0)}\left(\Delta \log \omega_{0}, \sum_{k \in \mathcal{N}} \Psi_{(k)} \Delta \log A_{k}+\sum_{f \in \mathcal{G}} \Psi_{(f)} \Delta \log \mathcal{L}_{f}^{\text {share }}\right)+\frac{1}{2} \mathbb{E}_{\lambda}\left(\frac{\phi}{1+\phi}(\Delta \log \lambda)^{2}\right),
\end{gathered}
$$

where the covariance and expectation on the last line are over factors $f \in \mathcal{G}$ and use initial factor shares $\lambda_{f}$ as probabilities and where $\Delta \log \lambda_{f}=\operatorname{Cov}_{\Omega^{(0)}}\left(\Delta \log \omega_{0}, \Psi_{(f)} / \lambda_{f}\right)$. 
The first line is the first-order approximation, capturing the direct effect of the shocks, as well as the endogenous reduction in factor supply. The first term accounts for the reduction in the supply of each factor when the share rather than the price of the factor is kept constant (recall that $\Delta \log \mathcal{L}_{f}^{\text {share }}=\left(1 /\left(1+\phi_{f}\right)\right) \Delta \log \mathcal{L}_{f}^{\text {price }}$ ). The second term captures the fact that overall factor supply is further reduced if the shares of more elastic factors decrease.

The second line is the second-order effect which captures nonlinearities. The first term on the second line captures the nonlinear effects of changes in the composition of demand and changes in the exogenous shocks. The final term captures the second-order effect of endogenous labor supply. For concreteness, focus on the case of negative factor supply shocks with $\Delta \log \mathcal{L}_{f}^{\text {share }} \leq 0$ for all $f \in \mathcal{G}$. The first-order term $\operatorname{Cov}_{\lambda}(\phi /(1+\phi), \Delta \log \lambda)$ shows that reductions in output are amplified if demand is redirected away (directly and indirectly) from more elastic factors and towards less elastic factors. The reduction in the supply of the former dominates the increase in the supply of the latter. The secondorder term $\frac{1}{2} \mathbb{E}_{\lambda}\left(\phi /(1+\phi)(\Delta \log \lambda)^{2}\right)$ is always positive and mitigates the reduction in output. This is because the endogenous reduction in the supply of a factor away from which demand is redirected comes together with a reduction in its share.

In contrast to our analysis with inelastic factor supplies, shocks to demand do have an effect on output even in the absence of other shocks. Indeed, the terms involving $\phi /(1+\phi)$,which reflect the endogenous response of factor supply, remain non-zero if the composition of demand changes, even if there are no other shocks.

Uniform elasticities and only shocks to factor supplies. We now give an illustrative example in the case where all the elasticities of substitution in production and in final demand are the same $\left(\theta_{j}=\theta\right.$ for all $\left.j \in 1+\mathcal{N}+\mathcal{G}\right)$. Changes in output can then be approximated at the first order by

$$
\begin{aligned}
& \Delta \log Y=\sum \lambda_{f} \Delta \log \mathcal{L}_{f}^{\text {share }} \\
& +\frac{\operatorname{Cov}_{\lambda}\left(\frac{1}{1-\frac{\phi}{1+\phi} \frac{\theta-1}{\theta}}, \Delta \log \mathcal{L}^{\text {share }}\right)}{\mathbb{E}_{\lambda}\left(\frac{\frac{1+\gamma}{1+\phi}}{1-\frac{\phi}{1+\phi} \frac{\theta-1}{\theta}}\right)}+\frac{\mathbb{E}_{\lambda}\left(\frac{\frac{\phi-\gamma}{1+\phi}}{1-\frac{\phi}{1+\phi} \frac{\theta-1}{\theta}}\right) \sum \lambda_{f} \Delta \log \mathcal{L}_{f}^{\text {share }}}{\mathbb{E}_{\lambda}\left(\frac{\frac{1+\gamma}{1+\phi}}{1-\frac{\phi}{1+\phi} \frac{\theta-1}{\theta}}\right)},
\end{aligned}
$$

where the covariance and expectation are over factors $f \in \mathcal{G}$ and use initial factor shares $\lambda_{f}$ as probabilities. The first line on the right-hand side is simply Hulten's theorem applied to the fixed-share labor supply shocks $\Delta \log \mathcal{L}_{f}^{\text {share }}$. It already contains the endogenous reduction in factor supplies when shares rather than prices are held constant, and 
when income is held constant.

The second line contains two terms capturing the effects of endogenous changes in factor supplies arising from changes in factor shares (first term) and income (second term). To unpack endogenous changes in factor supplies. Focus on the case of negative factor supply shocks with $\Delta \log \mathcal{L}_{f}^{\text {share }} \leq 0$ for all $f \in \mathcal{G}$.

We start with the first term on the second line. With complementarities $(\theta<1)$ the reduction in output is larger if less elastic factors are hit with larger negative $\Delta \log \mathcal{L}^{\text {share }}$ shocks. Factors hit with larger shocks increase their income shares and those hit with smaller shocks reduce their income shares. The change in the income shares triggers endogenous changes in supply, and the resulting endogenous reductions in the supplies of the latter dominate the endogenous increases in the supplies of the former if the latter are more elastic than the former. These effects reinforce themselves, which explains the denominator. The results are reversed with substituabilities $(\theta>1)$.

We now turn to the second term on the second line. There is a further reduction in factor supplies if substitution elasticities $\phi_{f}$ are on average greater than income elasticities $\gamma_{f}$ because then income effect does not compensate the substitution effect on average.

Consider the following two concrete examples. In the first example, imagine that the factors fall into two groups: capitals $\mathcal{K}$ and labors $\mathcal{W}$. To capture frictions in reallocation in the short run, imagine that the different capitals cannot be reallocated one into the other, and similarly for the labors. We assume that the supply of each of the labor is inelastic but that the utilization of each of the capitals is endogenous with an elasticity $\phi>0$ to its marginal product (but zero to income). We denote the total share of all the capitals by $\lambda_{\mathcal{K}}$ and the total share of all the labors by $\lambda_{\mathcal{W}}=1-\lambda_{\mathcal{K}}$. We hit this economy with negative shocks to the supplies of the labors $\Delta \log \mathcal{L}_{f}^{\text {price }}=\Delta \log \mathcal{L}_{f}^{\text {share }}<0$ for $f \in \mathcal{W}$ but we do not shock the capitals. Changes in output are given at the first order by

$$
\Delta \log Y \approx\left(1+\frac{\lambda_{\mathcal{K}} \phi}{\theta+\left(1-\lambda_{\mathcal{K}}\right) \phi}\right) \sum \lambda_{f \in \mathcal{L}} \Delta \log \mathcal{L}_{f}^{\text {price }}
$$

Hence endogenous capital utilization amplifies the reduction in output from the negative labor supply shocks. The effect is stronger when the elasticity of capital utilization $\phi$ is higher, which is intuitive. The effect is also stronger when the elasticity of substitution $\theta$ in production and in final demand is lower. This is because the negative shock to labor lowers the marginal product of capital more when $\theta$ is lower. In particular, this means that this effect is stronger with complementarities $(\theta<1)$ than with substituabilities $(\theta>1)$.

In the second example, imagine that there are negative labor supply shocks due to confinement efforts in a pandemic but no negative oil supply shocks. Assume that oil 
supply is less elastic than labor supplies in the short run but more elastic in the long run. Then the share of oil drops because demand falls more than supply, more so in the short run than in the long run. Similarly, the supply of oil drops, more so in the long run than in the short run. As a result, output drops more in the long run than in the short run.

\section{Welfare}

In this section, we turn our attention to welfare. Output as measured by real GDP is not necessarily a good measure of welfare for many reasons. ${ }^{11}$ We focus on the issue of shocks to the composition of final demand, which matters a great deal in the context of a global pandemic. With shocks to final demand, changes in real GDP do not reveal changes in welfare, absent all the other usual reasons why welfare and real GDP are not the same.

For example, if shocks to the composition of demand reflect changes in preferences, welfare must be evaluated through a compensating variation exercise under the postshock preferences as explained in Section 2.5. On the other hand, if shocks to the composition of demand are induced by policy, say the government forces households to consume a suboptimal basket, then the policy instruments represent distortions that lead to different changes in welfare and output. ${ }^{12}$

There are of course other (well-studied) issues that loosen the link between real GDP and welfare, but we do not focus on them. These are: (1) real GDP ignores distributional issues, ${ }^{13}$ (2) real GDP does not measure non-market activity like leisure and home production; ${ }^{14}$ (3) real GDP does not adequately capture the impact of new goods, disap-

\footnotetext{
${ }^{11}$ If we focus on a single period and put investments in final demand, we do not have a direct welfare interpretation of the object that we call welfare, and we must instead interpret it as the compensating variation of the post-shock final-demand quantity index, which coincides with its change between the pre-shock allocation and the post-shock allocation. This informative object is arguably what real GDP should seek to measure but only measures when there are no shocks to demand, and it can be characterized. If we focus on multiple periods at once and treat investments as intermediate goods using the model and results with an enriched set of commodities indexed by dates and states as explained in Section 2.2, we have a direct welfare interpretation, but the notion of real GDP that we compare it to is an inter-temporal Divisa index of consumptions at different dates and states (we explained above how to compute real GDP within a given period in the context of that model).

${ }^{12}$ These welfare calculation do not incorporate the benefits of policy interventions that correct for externalities.

${ }^{13}$ One imperfect way of dealing with these issues is to assume that the across-household transfers engineered by the tax system are the result of welfare maximization and hence represent compensating transfers. As long as preferences across households are homogenous, our propagation equations are not affected by these transfers, and our analysis in this section continues to apply.

${ }^{14}$ In principle, with appropriate data, one could come up with an extended measure of real GDP that encompasses these activities. We could then apply our results for real GDP to this extended measure and our corrections to get welfare.
} 
pearing goods, and quality adjustments. These difficult issues are well understood in the literature, and are all very relevant in the context of the Covid crisis, but we do not have anything to contribute about them in this paper.

\subsection{Comparative Statics for Welfare}

Our demand shifters $\omega_{0 k} / \bar{\omega}_{0 k}=\exp \left(\Delta \log \omega_{0 k}\right)$ are isomorphic to subsidy wedges between transacted prices $p_{k}$ and perceived prices $p_{k} /\left(\omega_{0 k} / \bar{\omega}_{0 k}\right)$, starting from a situation with no wedges. In this section, we find it convenient to embrace that interpretation. The idea is that behavior is shaped not only by transacted prices but also by shadow prices. The wedges could reflect the private internalization of some side effects associated with the final use of the different goods or the policy interventions through taxes and quantity restrictions.

The distinction between preference-driven and policy-induced changes in behavior is irrelevant for real GDP but it matters a great deal for welfare. To capture this distinction, we assume that the wedges can be decomposed accordingly. This means that we assume that $\Delta \log \omega_{0 k}=\Delta \log \omega_{0 k}^{\text {pref }}+\Delta \log \omega_{0 k}^{\text {pol }}$, where $\Delta \log \omega_{0 k}^{\text {pref }}$ and $\Delta \log \omega_{0 k}^{\text {pol }}$ capture respectively preference-driven and policy-induced changes in behavior. We impose the normalizations $\sum_{k \in \mathcal{N}} \bar{\Omega}_{0 k} \Delta \log \omega_{0 k}^{\text {pref }}=0$ and $\sum_{k \in \mathcal{N}} \bar{\Omega}_{0 k} \Delta \log \omega_{0 k}^{\mathrm{pol}}=0$.

Welfare. We compute changes in welfare as compensating variation by evaluating how much income consumers would have to give up to reach their initial utility under the post-shock prices and preferences. Because post-shock preferences are homothetic, this can be computed as the change in the corresponding quantity index between the preshock allocation and the post-shock allocation. Under the post-shock preferences, subsidies at the end point are $\Delta \log \omega_{0 k}^{\text {pol }}$ (policy distortions) and subsidies at the start point are $-\Delta \log \omega_{0 k}^{\text {pref }}$ (mis-optimization). Equivalently, taxes at the end point are $-\Delta \log \omega_{0 k}^{\text {pol }}$ and taxes at the start point are $\Delta \log \omega_{0 k}^{\text {pref }}$. We refer the reader to Appendix A.2 for more details and for a proof of the following proposition.

Proposition 6. Changes in welfare can be approximated up to the second order by

$$
\Delta \log W \approx \Delta \log Y+\frac{1}{2} \sum_{k \in \mathcal{N}} \Omega_{0 k} \Delta \log y_{0 k}\left(\Delta \log \omega_{0 k}^{p r e f}-\Delta \log \omega_{0 k}^{p o l}\right),
$$


where

$$
\begin{aligned}
\frac{1}{2} \sum_{k \in \mathcal{N}} \Omega_{0 k} \Delta \log y_{0 k}\left(\Delta \log \omega_{0 k}^{p r e f}-\Delta \log \omega_{0 k}^{p o l}\right) \approx \\
+\frac{1}{2} \theta_{0} \operatorname{Cov}_{\Omega^{(0)}}\left(\Delta \log \omega_{0}^{p r e f}-\Delta \log \omega_{0}^{p o l}, \sum_{k \in \mathcal{N}} \Psi_{(k)} \Delta \log A_{k}+\sum_{f \in \mathcal{G}} \Psi_{(f)} \Delta \log L_{f}\right) \\
\quad-\frac{1}{2} \theta_{0} \operatorname{Cov}_{\Omega^{(0)}}\left(\Delta \log \omega_{0}^{p r e f}-\Delta \log \omega_{0}^{p o l}, \sum_{k \in \mathcal{N}} \Psi_{(k)} \Delta \log \lambda_{k}+\sum_{f \in \mathcal{G}} \Psi_{(f)} \Delta \log \lambda_{f}\right) \\
\quad+\frac{1}{2} \theta_{0}\left(\operatorname{Var}_{\Omega^{(0)}}\left(\Delta \log \omega^{p r e f}\right)-\operatorname{Var}_{\Omega^{(0)}}\left(\Delta \log \omega^{p o l}\right)\right),
\end{aligned}
$$

where changes in output $\Delta \log Y$, sales and factor shares $\Delta \log \lambda_{i}$ and $\Delta \log \lambda_{f}$ are given by Proposition 1 and Corollary 1.

Starting with the first expression, we see that changes in welfare coincide with changes in output at the first order. The corrective terms are zero when there are no shock to demand $\left(\Delta \log \omega_{0 k}^{\text {pref }}=\Delta \log \omega_{0 k}^{\text {pol }}=0\right.$ for all $\left.k \in 1+\mathcal{N}\right)$, and so in this case, changes in welfare and changes in output coincide at the second order, and in fact at all orders. But these terms are not zero at the second order when there are shock to demand. In this case, there are different nonlinearities in output and in welfare captured by the second term $(1 / 2) \sum_{k \in \mathcal{N}} \Omega_{0 k} \Delta \log y_{0 k}\left(\Delta \log \omega_{0 k}^{\text {pref }}-\Delta \log \omega_{0 k}^{\text {pol }}\right)$ on the right-hand side of the first expression. Each term $k$ in this sum is the Harberger triangle associated with the tax on good $k$ under the post-shock preferences. Here $(1 / 2)\left(\Delta \log \omega_{0 k}^{\text {pref }}-\Delta \log \omega_{0 k}^{\text {pol }}\right)$ is the average tax on good $k$ along the path of the shocks, $\Delta \log y_{0 k}$ is the change in the final consumption of good $k$ along the path of the shocks, and $\Omega_{0 k}$ is the share of final good $k$.

The second expression expresses the sum of these Harberger triangles as function of the shocks and of changes in sales and expenditure shares. The first two terms on the right-hand side capture the different interactions of shocks to demand and to productivities and factor supplies in output and in welfare. The third term captures the different independent effect of shocks to demand in output and welfare.

Consider for example the case when there are shocks to demand but no shocks to productivities or to factor supplies, the first two terms are zero and we are left with the third term $(1 / 2) \theta_{0}\left(\operatorname{Var}_{\Omega^{(0)}}\left(\Delta \log \omega_{0}^{\text {pref }}\right)-\operatorname{Var}_{\Omega^{(0)}}\left(\Delta \log \omega_{0}^{\text {pol }}\right)\right)$. If the shock to demand is a pure shock to preferences $\left(\Delta \log \omega_{0 k}^{\text {pol }}=0\right.$ for all $\left.k \in \mathcal{N}\right)$, then this term is positive. In this case, changes in welfare $\Delta \log W>0$ reflect the gains from re-optimizing consumption under the new preferences starting from a sub-optimal initial allocation. If the shock to demand is entirely induced by policy $\left(\Delta \log \omega_{0 k}^{\text {pref }}=0\right.$ for all $\left.k \in \mathcal{N}\right)$, then this term 
is negative. In this case, changes in welfare $\Delta \log W<0$ reflect the misallocation losses induced by the policy distortions. In both cases, there are no changes in output $(\Delta \log Y=$ $0)$ but there are changes in welfare $(\Delta \log W \neq 0)$.

Price deflators. This analysis also has dual implications for price deflators. It implies that the GDP deflator and the CPI are poor guides to welfare when there are shock to demand. For example, changes in the GDP deflator $\Delta \log P=\Delta \log G D P-\Delta \log Y$ coincide with changes in the welfare deflator $\Delta \log P^{W}=\Delta \log G D P-\Delta \log W$ only when there are no shocks to demand.

\subsection{Illustrative Example}

We derive a fully-worked out example illustrating the difference between output and welfare in a Cobb-Douglas economy. We refer the reader to Appendix A.2 for another fully-worked out example with acyclic economies.

Suppose that all the elasticities of substitution in production and in final demand are equal to one $\left(\theta_{j}=1\right.$ for all $\left.j \in 1+\mathcal{N}\right)$. Then we already saw in Proposition 3 that changes in output can be approximated to the second order by

$$
\begin{aligned}
\Delta \log Y \approx \sum_{k \in \mathcal{N}} \lambda_{k} \Delta \log A_{k}+\sum_{f \in \mathcal{G}} \lambda_{f} \Delta \log L_{f} \\
+\frac{1}{2} \operatorname{Cov}_{\Omega^{(0)}}\left(\Delta \log \omega_{0}^{\text {pref }}, \sum_{k \in \mathcal{N}} \Psi_{(k)} \Delta \log A_{k}+\sum_{f \in \mathcal{G}} \Psi_{(f)} \Delta \log L_{f}\right) .
\end{aligned}
$$

We now turn to changes in welfare. If the shocks to demand are entirely induced by policy, changes in welfare can be approximated up to the second order by

$$
\Delta \log W \approx \Delta \log Y-\frac{1}{2} \sum_{f \in \mathcal{G}} \lambda_{f} \operatorname{Var}_{\Xi(f)}\left(\Delta \log \omega_{0}\right)
$$

where $\Xi$ is the $\mathcal{G} \times(1+\mathcal{N}+\mathcal{G})$ matrix with $f i$-th element $\Xi_{i f}=\Omega_{0 i} \Psi_{i f} / \lambda_{f}$ which measure the fraction of the income share of factor $f$ coming directly and indirectly from spending on good $i$ in final demand. Hence for a given factor $f$, the term $\operatorname{Var}_{\Xi(f)}\left(\Delta \log \omega_{0}\right)$ is the variance of the different shocks to demand $\Delta \log \omega_{0 k}$ using $\Xi_{k f}$ the probability measure. In this case, changes in welfare discount entirely the interactions between shocks to demand and shocks to productivities and factor supplies. For example, reallocation of demands toward expanding factors increases output but does not increase welfare because 
these reallocations are wasteful since they reflect distortions. As already discussed above, changes in welfare also feature independent losses from shocks to demand.

If instead the shocks to demand are pure preference shocks, changes in welfare can be approximated up to the second order by

$$
\begin{aligned}
\Delta \log W \approx \Delta \log Y+ & +\frac{1}{2} \sum_{g \in \mathcal{G}} \lambda_{g} \operatorname{Var}_{\Xi(g)}\left(\Delta \log \omega_{0}\right) \\
& +\frac{1}{2} \operatorname{Cov}_{\Omega^{(0)}}\left(\Delta \log \omega_{0}^{\text {pref }}, \sum_{k \in \mathcal{N}} \Psi_{(k)} \Delta \log A_{k}+\sum_{f \in \mathcal{G}} \Psi_{(f)} \Delta \log L_{f}\right) .
\end{aligned}
$$

In this case, changes in welfare count the interactions between shocks to demand and shocks to productivities and factor supplies twice compared to changes in output. For example, reallocation of demands toward expanding factors increases welfare twice as much as output because changes in welfare use stable post-shock preferences. As already discussed above, changes in welfare also feature independent gains from shocks to demand.

\section{Quantitative Application}

For our quantitative experiment, we use an input-output model to capture a stylized version of the U.S. economy. We hit this economy with different labor supply shocks and analyze the response of aggregate output and welfare. At present, there is no data in how the composition of final demand changed due to the shock. In the next revision of the paper, once this data has become available, we also quantitatively investigate the importance of changes in the composition of final demand and productivity shocks for both welfare and real GDP.

\subsection{Setup}

Calibrating the economy. There are 66 industries and industrial production functions combine labor, capital, and intermediates. The share parameters of the functions are calibrated so that at the initial pre-shock allocation, expenditure shares match those in the input-output tables provided by the BEA. We focus on the short run and assume that labor and capital cannot be reallocated across sectors.

The nesting structure is the following. In each sector, labor and capital are combined a value-added nest with an elasticity $\eta$, intermediates are combined in an intermediates 
nest with an elasticity $\theta$, these two nests are then combined in a sector nest with an elasticity $\epsilon$, and final consumptions are combined in a final-demand nest with an elasticity $\sigma$. We therefore allow for differences in the elasticities, but we do not allow them to vary by sector, because such disaggregated estimates are not available.

We construct the input-output matrix using the annual U.S. input-output data from the BEA, dropping the government, noncomparable imports, and second-hand scrap industries. The dataset contains industrial output and inputs for 66 industries.

Based on the empirical literature, there is considerable uncertainty as to what the right values for these elasticities should be. Atalay (2017), Herrendorf et al. (2013), and Oberfield and Raval (2014), all of use an elasticity of substitution in consumption $\sigma$ slightly less than one. Atalay (2017) estimates the elasticity of substitution across intermediates and value added $\epsilon$ to be between 0.4 and 0.8 and Boehm et al. (2019), who estimate this elasticity to be close to 0 . Atalay (2017) estimates the elasticity of substitution across intermediate inputs to be $\theta=0.001$. Finally the typical estimates for the elasticity of substitution between capital and labor $\eta$ range between 0.3 and 1 .

Not all these elasticities are estimated at the same horizon, and this matters since we can expect elasticities to be lower in the short run than in the medium run. For instance, Boehm et al. (2019), who estimate short-run elasticities, find lower estimates than studies that use long-run data.

To illustrate the range of plausible possibilities, we consider three calibrations: a calibration with very low elasticities $(\sigma, \theta, \epsilon, \eta)=(0.5,0.001,0.3,0.2)$; a calibration with low elasticities $(\sigma, \theta, \epsilon, \eta)=(0.7,0.001,0.3,0.2)$; and a calibration with higher elasticities $(\sigma, \theta, \epsilon, \eta)=(0.95,0.001,0.7,0.5)$. We think of them as being relevant in the very short run, the short run, and the medium run. We also report the results for a log-linear CobbDouglas economy with $(\sigma, \theta, \epsilon, \eta)=(1,1,1,1)$ where Hulten's theorem holds exactly and not just as a first-order approximation.

Calibrating the shocks. We consider shocks to labor supplies. The shock is a reduction in labor supplies calibrated to match the changes in hours worked by sector from the May 2020 BLS Economic News release. There are concerns that these numbers underestimated the actual reductions that took place because the underlying survey may not appropriately classify furloughed or absent employees as temporarily unemployed. To allow for this possibility, we consider different scenarios which multiply the log reduction in hours by industry, reported by the BLS, by a scaling factor $x \in[0,1.5]$. We refer to $x$ as the size of the shock. As mentioned before, when $x=1$, aggregate hours falls by $13 \%$, whereas when $x=1.5$, aggregate hours falls by $20 \%$. For concreteness, we focus our 
attention on two scenarios: in the less extreme scenario, we assume that in each sector, the reduction in hours worked coincides with that given by the BLS, so that $x=1$; in the more extreme scenario, we inflate the reduction in hours worked in each sector reported by the BLS by $50 \%$, so that $x=1.5$.

\begin{tabular}{rrrrr}
\hline Scenario & Very short run & Short run & Medium run & Cobb-Douglas \\
\hline Less extreme $(x=1)$ & $12 \%$ & $11 \%$ & $10 \%$ & $9 \%$ \\
More extreme $(x=1.5)$ & $28 \%$ & $15 \%$ & $17 \%$ & $14 \%$ \\
\hline \hline
\end{tabular}

Table 1: Output reduction as a function of the size of the labor supply shock $x$.

Table 1 displays the response of output for our three benchmark scenarios (short-run or medium-run) depending on the elasticities of substitution (lower or higher), as well as the log-linear first-order approximation implied by Hulten's theorem (equivalently, a Cobb-Douglas model). Figure 5 displays the same results as Table 1 but explores a wider range of scenarios. The Cobb-Douglas economy serves as a yardstick to measure nonlinearities: nonlinearities account for the reductions in output beyond those of the Cobb-Douglas economy.

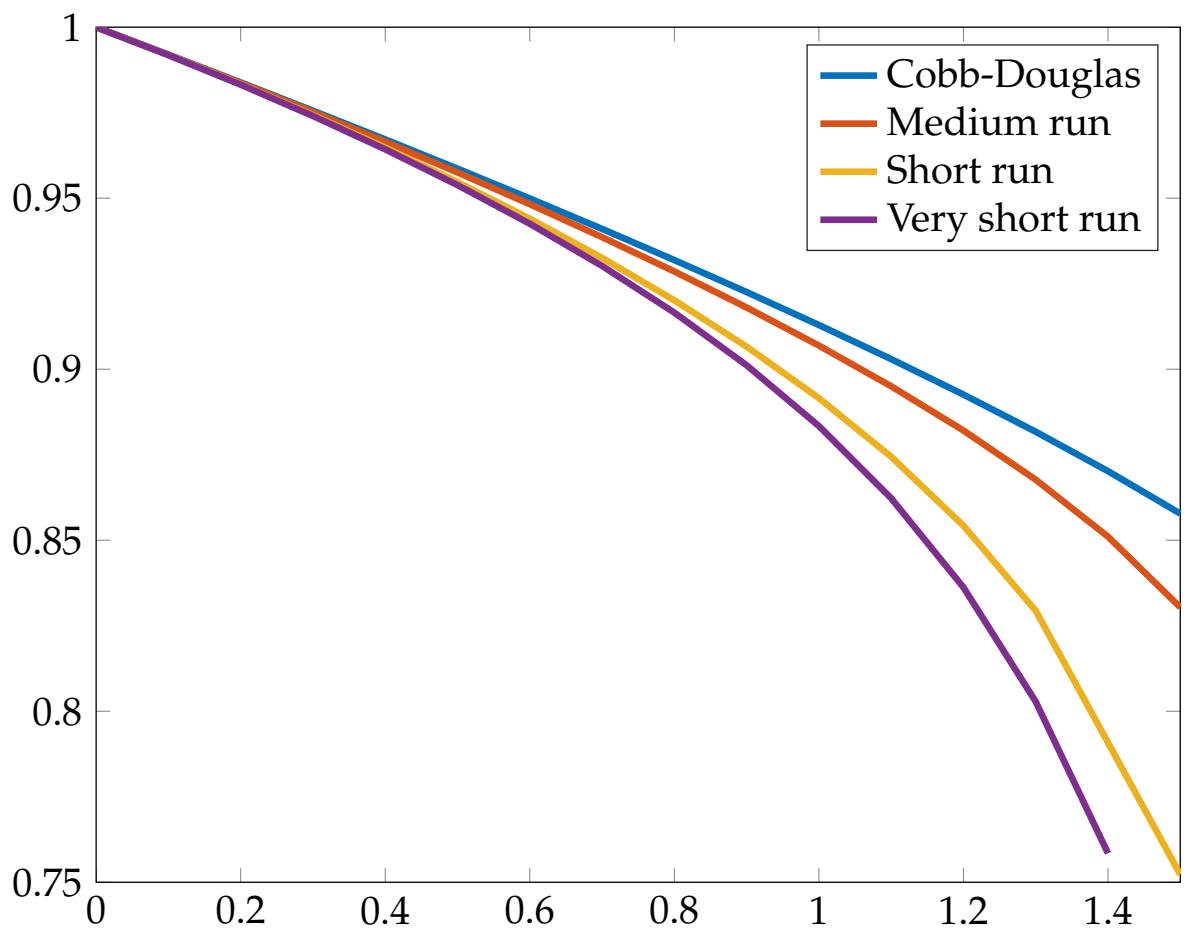

Figure 5: Output change $\Delta Y / \bar{Y}$ as a function of the size of the shock $x$.

In the more extreme scenario, output drops by $14 \%$ in the Cobb-Douglas economy, 
by $17 \%$ in the economy with higher elasticities, by $25 \%$ in the economy with low elasticities, and by $28 \%$ in the economy with very low elasticities. In this case, nonlinearities considerably amplify the impact of the shock on output.

In the less extreme scenario, output drops by $9 \%$ in the Cobb-Douglas economy, by $10 \%$ in the economy with higher elasticities, by $11 \%$ in the economy with low elasticities, and by $12 \%$ in the economy with very low elasticities. Nonlinearities significantly amplify the impact of the shock on output, although less than in the more extreme scenario.

In both scenarios, and for all sets of elasticities, nonlinearities play an important role in amplifying the reduction in output. That nonlinear amplification is greater in the more extreme scenario is intuitive since nonlinearities matter more for larger shocks. That nonlinear amplification is greater with lower elasticities is intuitive since nonlinear amplification of negative shocks increases with the degree of complementarities in the economy.

At the time of writing, most forecasts for the reduction in output cluster around a reduction of $12 \%$. This number falls squarely in the range of the predictions of our model, and could reflect a significant amount of nonlinear amplification. However, the reduction in output in the data is likely to under-estimate the true decline in output. One reason is because the GDP deflator is likely under-estimated as many goods became unavailable during the period of March-April. One way to think about it is that the prices of these goods increased to infinity.Because these price increases will not be recorded in the GDP deflator, the change in the GDP deflator will be biased downwards, and, as a result, the change in real GDP will be biased upwards. Another reason why the change in real GDP may be biased downwards is that it the change in the GDP deflator is not computed in continuous time but as a discrete-time approximation. It is therefore entirely possible that "true" real GDP, measured in continuous time, is a lot lower than the official number suggests. The more sudden is the shock, the worse is the discrete time approximation to the continuous time change, and the more room there is for nonlinear amplification.

\section{Conclusion}

This paper analytically characterizes and numerically quantifies the importance of nonlinearities. We show that in the presence of nonlinear production and consumption functions, heterogeneous negative supply shocks can be very costly to aggregate output. Our results suggest that the heterogeneous nature of the shock likely significantly compounds its negative effects. Large shocks to some sectors drag down other sectors with them. Changes in the composition of final demand, depending on whether they increase or reduce spending on negatively affected sectors directly and directly through their supply 
chains, can further amplify or mitigate these nonlinearities. In future versions of the paper, as more data becomes available, we hope to revise these numbers to better reflect reality.

\section{References}

Acemoglu, Daron, Victor Chernozhukov, Iván Werning, and Michael D. Whinston, “A Multi-Risk SIR Model with Optimally Targeted Lockdown,” Technical Report 2020.

Alvarez, Fernando E, David Argente, and Francesco Lippi, “A simple planning problem for covid-19 lockdown," Technical Report, National Bureau of Economic Research 2020.

Atalay, Enghin, "How important are sectoral shocks?," American Economic Journal: Macroeconomics, 2017, 9 (4), 254-80.

Atkeson, Andrew, “How Deadly Is COVID-19? Understanding The Difficulties With Estimation Of Its Fatality Rate," Technical Report, National Bureau of Economic Research 2020.

_ , "What will be the economic impact of COVID-19 in the US? Rough estimates of disease scenarios," Technical Report, National Bureau of Economic Research 2020.

Baqaee, David and Emmanuel Farhi, "A Short Note on Aggregating Productivity," NBER Working Papers 25688, National Bureau of Economic Research, Inc March 2019.

_ and _ , "The Macroeconomic Impact of Microeconomic Shocks: Beyond Hulten's Theorem," Econometrica (Forthcoming), 2019.

_ and _ , "Keynesian Production Networks with an Application to the Covid-19 Crisis," Technical Report 2020.

Barro, Robert J, José F Ursúa, and Joanna Weng, "The coronavirus and the great influenza pandemic: Lessons from the "spanish flu" for the coronavirus's potential effects on mortality and economic activity," Technical Report, National Bureau of Economic Research 2020.

Barrot, Jean-Noel, Basile Grassi, and Julien Sauvagnat, "Sectoral effects of social distancing," Available at SSRN, 2020. 
Berger, David W, Kyle F Herkenhoff, and Simon Mongey, "An SEIR infectious disease model with testing and conditional quarantine," Technical Report, National Bureau of Economic Research 2020.

Bethune, Zachary A and Anton Korinek, "Covid-19 Infection Externalities: Trading Off Lives vs. Livelihoods," Technical Report, National Bureau of Economic Research 2020.

Bigio, Saki, Mengbo Zhang, and Eduardo Zilberman, “Transfers vs Credit Policy," Technical Report 2020.

Bodenstein, Martin, Giancarlo Corsetti, and Luca Guerrieri, "Social Distancing and Supply Disruptions in a Pandemic," 2020.

Boehm, Christoph E, Aaron Flaaen, and Nitya Pandalai-Nayar, "Input linkages and the transmission of shocks: Firm-level evidence from the 2011 Tōhoku earthquake," Review of Economics and Statistics, 2019, 101 (1), 60-75.

Bonadio, Barthélémy, Zhen Huo, Andrei A. Levchenko, and Nitya Pandalai-Nayar, "Global Supply Chains in the Pandemic," Technical Report 2020.

Caballero, Ricardo J and Alp Simsek, "A Model of Asset Price Spirals and Aggregate Demand Amplification of a'COVID-19'Shock," Available at SSRN 3576979, 2020.

Cakmakli, Cem, Selva Demiralp, Sebnem Kalemli-Ozcan, Sevcan Yesiltas, and Muhammed A Yildirim, "COVID-19 and Emerging Markets: An Epidemiological Multi-Sector Model for a Small Open Economy with an Application to Turkey," Working Paper 27191, National Bureau of Economic Research May 2020.

Carvalho, Vasco M and Alireza Tahbaz-Salehi, "Production networks: A primer," Annual Review of Economics, 2019, 11, 635-663.

Christiano, Lawrence J, Martin Eichenbaum, and Charles L Evans, "Nominal rigidities and the dynamic effects of a shock to monetary policy," Journal of political Economy, 2005, $113(1), 1-45$.

Dingel, Jonathan I. and Brent Neiman, "How Many Jobs Can be Done at Home?," NBER Working Papers 26948, National Bureau of Economic Research, Inc April 2020.

e Castro, Miguel Faria, “Fiscal policy during a Pandemic,” Technical Report 2020.

Eichenbaum, Martin S, Sergio Rebelo, and Mathias Trabandt, "The macroeconomics of epidemics," Technical Report, National Bureau of Economic Research 2020. 
${ }_{-},{ }_{-}$, and _ , "The macroeconomics of testing during epidemics," Technical Report 2020.

Fernández-Villaverde, Jesús and Charles I Jones, "Estimating and Simulating a SIRD Model of COVID-19 for Many Countries, States, and Cities."

Fornaro, Luca and Martin Wolf, "Covid-19 coronavirus and macroeconomic policy," 2020.

Galle, Simon, Andres Rodriguez-Clare, and Moises Yi, "Slicing the pie: Quantifying the aggregate and distributional effects of trade," Technical Report, National Bureau of Economic Research 2017.

Glover, Andrew, Jonathan Heathcote, Dirk Krueger, and José-Víctor Ríos-Rull, "Health versus wealth: On the distributional effects of controlling a pandemic," 2020.

Gourinchas, Pierre-Olivier, "Flattening the pandemic and recession curves," Mitigating the COVID Economic Crisis: Act Fast and Do Whatever, 2020, p. 31.

_, Sebnem Kalemli-Ozcan, Veronika Penciakova, and Nick Sander, "COVID-19 an Business Failures," Working Paper, Berkeley May 2020.

Guerrieri, Veronica, Guido Lorenzoni, Ludwig Straub, and Iván Werning, "Macroeconomic Implications of COVID-19: Can Negative Supply Shocks Cause Demand Shortages?," Technical Report, National Bureau of Economic Research 2020.

Hall, Robert E, Charles I Jones, and Peter J Klenow, "Trading off consumption and covid-19 deaths," Technical Report, Working Paper 2020.

Herrendorf, Berthold, Richard Rogerson, and Akos Valentinyi, "Two perspectives on preferences and structural transformation," American Economic Review, 2013, 103 (7), 2752-89.

Hulten, Charles R, "Growth accounting with intermediate inputs," The Review of Economic Studies, 1978, pp. 511-518.

Jones, Callum J, Thomas Philippon, and Venky Venkateswaran, "Optimal mitigation policies in a pandemic: Social distancing and working from home," Technical Report, National Bureau of Economic Research 2020.

Jones, Charles I, "Intermediate goods and weak links in the theory of economic development," American Economic Journal: Macroeconomics, 2011, pp. 1-28. 
Jorda, Oscar, Sanjay R Singh, and Alan M Taylor, "Longer-run economic consequences of pandemics," Technical Report, National Bureau of Economic Research 2020.

Kaplan, Greg, Benjamin Moll, and Gianluca Violante, "Pandemics according to HANK," 2020.

Krueger, Dirk, Harald Uhlig, and Taojun Xie, "Macroeconomic dynamics and reallocation in an epidemic," 2020.

McKenzie, Lionel W, "On the existence of general equilibrium for a competitive market," Econometrica: journal of the Econometric Society, 1959, pp. 54-71.

Mongey, Simon, Laura Philosoph, and Alex Weinberg, "Which Workers Bear the Burden of Social Distancing Policy?," University of Chicago Working Paper 2020.

Oberfield, Ezra and Devesh Raval, "Micro Data and Macro Technology," Working Paper 20452, National Bureau of Economic Research September 2014. 


\section{A Appendix}

\section{A.1 Proofs}

Proposition 1. We use Hulten's theorem

$$
d \log Y_{s}=\sum_{k \in \mathcal{N}} \lambda_{k, s} d \log A_{k, s}+\sum_{f \in \mathcal{G}} \lambda_{f, s} d \log L_{f, s}
$$

Integrating to get

$$
\Delta \log Y=\int d \log Y_{s}
$$

we get

$$
\Delta \log Y=\sum_{k \in \mathcal{N}} \int \lambda_{k, s} d \log A_{k, s}+\sum_{f \in \mathcal{G}} \int \lambda_{f, s} d \log L_{f, s}
$$

$$
\begin{aligned}
\Delta \log Y=\sum_{k \in \mathcal{N}} \lambda_{k, \bar{s}} \int d \log & A_{k, s}+\sum_{f \in \mathcal{G}} \lambda_{f, \bar{s}} \int d \log L_{f, s} \\
& +\sum_{k \in \mathcal{N}} \int\left(\lambda_{k, s}-\lambda_{k, \bar{s}}\right) d \log A_{k, s}+\sum_{f \in \mathcal{G}} \int\left(\lambda_{f, s}-\lambda_{f, \bar{s}}\right) d \log L_{f, s},
\end{aligned}
$$

$$
\begin{aligned}
& \Delta \log Y \approx \sum_{k \in \mathcal{N}} \lambda_{k, \bar{s}} \int d \log A_{k, s}+\sum_{f \in \mathcal{G}} \lambda_{f, \bar{s}} \int d \log L_{f, s} \\
& \quad+\left.\sum_{k \in \mathcal{N}} \lambda_{k, \bar{s}} \frac{d \log \lambda_{k, s}}{d s}\right|_{s=\bar{s}} \int(s-\bar{s}) d \log A_{k, s}+\left.\sum_{f \in \mathcal{G}} \lambda_{f, \bar{s}} \frac{d \log \lambda_{f, s}}{d s}\right|_{s=\bar{s}} \int(s-\bar{s}) d \log L_{f, s}
\end{aligned}
$$

$$
\begin{aligned}
\Delta \log Y \approx \sum_{k \in \mathcal{N}} \lambda_{k} \Delta \log A_{k}+\sum_{f \in \mathcal{G}} & \lambda_{f} \Delta \log L_{f} \\
& +\frac{1}{2} \sum_{k \in \mathcal{N}} \lambda_{k} \Delta \log \lambda_{k} \Delta \log A_{k}+\frac{1}{2} \sum_{f \in \mathcal{G}} \lambda_{f} \Delta \log \lambda_{f} \Delta \log L_{f} .
\end{aligned}
$$

\section{A.2 Welfare in Section 5}

To compute welfare, we find it more convenient to think of demand shocks as shocks $\Delta \log \omega_{0 k}$ to subsidy wedges transacted prices $p_{k}$ and perceived prices $p_{k} / \omega_{0 k}$ with $\bar{\omega}_{0 k}=$ 1. Changes in these subsidy wedges $\Delta \log \omega_{0 k}=\Delta \log \omega_{0 k}^{\text {pref }}+\Delta \log \omega_{0 k}^{\text {pol }}$ reflect changes in preferences and government interventions captured respectively by $\Delta \log \omega_{0 k}^{\text {pref }}$ and 
$\Delta \log \omega_{0 k}^{\text {pol }}$. We impose the normalizations

$$
\sum_{k \in \mathcal{N}} \bar{\Omega}_{0 k} \Delta \log \omega_{0 k}^{\text {pref }}=\sum_{k \in \mathcal{N}} \bar{\Omega}_{0 k} \Delta \log \omega_{0 k}^{\text {pol }}=0
$$

We compute changes in welfare as changes in compensating variations. To do so, we think of the wedges as actual actual subsidies $\Delta \log \omega_{0 k}$ and remove the distinction between transacted and perceived prices. Because preferences are homothetic, we can compute compensating variation as the change in output along a path that starts ends at the start point with subsidies $-\Delta \log \omega_{0 k}^{\text {pref }}$, at ends at the end point with subsidies $\Delta \log \omega_{0 k}^{\text {pol }}$. Doing so ensure that we compute the change in a homothetic index corresponding to the post-shock preferences.

For example, when shocks to demand reflect changes in preferences, subsidies are $-\Delta \log \omega_{0 k}^{\text {pref }}$ at the start point because the initial allocation does not minimize expenditure given utility under the post-shock preferences (it minimizes expenditure under the pre-shock preferences), and subsidies are 0 at the end point because the final allocation minimizes expenditure under the post-shock preferences. Similarly, when shocks to demand are induced by policy, subsidies are 0 at the start point because the initial allocation minimizes expenditure given utility (since there are no policy distortions), and subsidies are $\Delta \log \omega_{0 k}^{\text {pol }}$ at the end point because the final allocation do not minimize expenditure (since there are distortions induced by policy).

We compute changes in output along this path using the formula in derived in Baqaee and Farhi (2019a) and get

$$
\begin{aligned}
& \Delta \log W \approx \sum_{i \in \mathcal{N}-\mathcal{G}} \lambda_{i} \Delta \log A_{i}+\sum_{f \in \mathcal{G}} \lambda_{f} \Delta \log L_{f} \\
&+\frac{1}{2} \sum_{i \in \mathcal{N}-\mathcal{G}} \lambda_{i} \Delta \log \lambda_{i} \Delta \log A_{i}+\frac{1}{2} \sum_{f \in \mathcal{G}} \lambda_{f} \Delta \log \lambda_{f} \Delta \log L_{f} \\
& \quad+\frac{1}{2} \sum_{k \in \mathcal{N}} \Omega_{0 k} \Delta \log y_{0 k}\left(\Delta \log \omega_{0 k}^{\text {pref }}-\Delta \log \omega_{0 k}^{\text {pol }}\right) .
\end{aligned}
$$

We then use

$$
\Delta \log y_{0 k}=\theta_{0}\left(\Delta \log \omega_{0 k}+\sum_{i \in \mathcal{N}-\mathcal{G}} \Psi_{k i} \Delta \log A_{i}-\sum_{f \in \mathcal{G}} \Psi_{k f}\left(\Delta \log \lambda_{f}-\Delta \log L_{f}\right)\right)
$$


Replacing and manipulating, we get

$$
\begin{aligned}
\Delta \log W \approx \sum_{i \in \mathcal{N}-\mathcal{G}} \lambda_{i} \Delta \log A_{i}+\sum_{f \in \mathcal{G}} \lambda_{f} \Delta \log L_{f} \\
+\frac{1}{2} \sum_{i \in \mathcal{N}-\mathcal{G}} \lambda_{i} \Delta \log \lambda_{i} \Delta \log A_{i}+\frac{1}{2} \sum_{f \in \mathcal{G}} \lambda_{f} \Delta \log \lambda_{f} \Delta \log L_{f} \\
+\frac{1}{2} \theta_{0} \operatorname{Cov}_{\Omega^{(0)}}\left(\Delta \log \omega_{0}^{\mathrm{pref}}-\Delta \log \omega_{0}^{\mathrm{pol}}, \sum_{i \in 1+\mathcal{N}} \Psi_{(i)} \Delta \log A_{i}+\sum_{f \in \mathcal{G}} \Psi_{(f)} \Delta \log L_{f}\right) \\
-\frac{1}{2} \theta_{0} \operatorname{Cov}_{\Omega^{(0)}}\left(\Delta \log \omega_{0}^{\mathrm{pref}}-\Delta \log \omega_{0}^{\mathrm{pol}}, \sum_{i \in 1+\mathcal{N}} \Psi_{(i)} \Delta \log \lambda_{i}+\sum_{f \in \mathcal{G}} \Psi_{(f)} \Delta \log \lambda_{f}\right) \\
\quad+\frac{1}{2} \theta_{0}\left(\operatorname{Var}_{\Omega^{(0)}}\left(\Delta \log \omega_{0}^{\mathrm{pref}}\right)-\operatorname{Var}_{\Omega^{(0)}}\left(\Delta \log \omega_{0}^{\mathrm{pol}}\right)\right),
\end{aligned}
$$

where recall that $\Delta \log \omega_{0 k}=\Delta \log \omega_{0 k}$.

\section{A.2.1 Additional Illustrative Example with Acyclic Networks}

Suppose now that the production network is acyclic. We already saw in Proposition 5 and exact expression for changes in output $\Delta \log Y$ when there are no shocks to demand, as well as a second-order approximation even when there are shocks to demand. We can strengthen the results for welfare and derive an exact recursive characterization from upstream to downstream even when there are shocks to demand:

$$
e^{\Delta \log W}=e^{\Delta \log y_{0}}=\left(\sum_{j \in u(i)} \bar{\Omega}_{i j} e^{\Delta \log \omega_{0 j}^{\text {pref }}}\left(e^{\Delta \log y_{j}}\right)^{\frac{\theta_{0}-1}{\theta_{0}}}\right)^{\frac{\theta_{0}}{\theta_{0}-1}},
$$

where and we have the recursion from upstream to downstream

$$
e^{\Delta \log y_{i}}=e^{\Delta \log A_{i}}\left(\sum_{j \in u(i)} \bar{\Omega}_{i j}\left(e^{\Delta \log y_{j}}\right)^{\frac{\theta_{i}-1}{\theta_{i}}}\right)^{\frac{\theta_{i}}{\theta_{i}-1}},
$$

for $i \neq 0$ and where $\Delta \log y_{i}=\Delta \log L_{f}$ if $i=f \in \mathcal{G}$ is a factor . We also have fully-solvedout approximations of changes in output to the second order using Proposition 6 and the first-order approximations for changes in sales and factor shares given in Appendix A.3.

In this case, the shocks do not lead to any reallocations of resources. Shocks to demand therefore have no independent impact on welfare. In the presence of other shocks, preference-driven shocks to demand only change the final-demand quantity index used 
to evaluate changes in final consumptions given by the shocks to productivities and factor supplies, and policy-driven shocks to demand have no impact. These results generalize to sub-networks with uniform elasticities embedded in acyclic networks.

\section{A.3 Expressions for Sales and Factor Shares in Section 3.3}

Proposition 3. We have exact and first-order approximations of changes in sales and factor shares:

$$
\bar{\lambda}_{i} \exp \left(\Delta \log \lambda_{i}\right)=\operatorname{Cov}_{\bar{\Omega}^{(0)}}\left(\Delta \log \omega_{0}, \bar{\Psi}_{(i)}\right)
$$

Proposition 4. We have exact and first-order approximations of changes in factor shares:

$$
\begin{gathered}
\bar{\lambda}_{f} e^{\Delta \log \lambda_{f}}=\frac{\bar{\lambda}_{f}\left(e^{\Delta \log L_{f}}\right)^{\frac{\theta-1}{\theta}}}{\sum_{f \in \mathcal{G}} \bar{\lambda}_{f}\left(e^{\Delta \log L_{f}}\right)^{\frac{\theta-1}{\theta}},} \\
\lambda_{f} \Delta \log \lambda_{f} \approx \frac{\theta-1}{\theta} \lambda_{f}\left(\Delta \log L_{f}-\mathbb{E}_{\lambda}(\Delta \log L)\right) .
\end{gathered}
$$

Proposition 5. We have exact expressions for sales and factor shares as a recursion from downstream to upstream

$$
\bar{\lambda}_{i} e^{\Delta \log \lambda_{i}}=\bar{\lambda}_{d(i)} e^{\Delta \log \lambda_{d(i)}} \frac{\bar{\Omega}_{d(i) i} e^{\delta_{0 d(i)} \theta_{0} \Delta \log \omega_{0 i}}\left(e^{\Delta \log y_{i}}\right)^{\frac{\theta_{d(i)}-1}{\theta_{d(i)}}}}{\sum_{j \in d(i)} \bar{\Omega}_{d(i) j} e^{\delta_{0 d(i)} \theta_{0} \Delta \log \omega_{0 j}}\left(e^{\Delta \log y_{j}}\right)^{\frac{\left.\theta_{d(i)}\right)^{-1}}{\theta_{d(i)}}}},
$$

where by definition $\Delta \log \lambda_{0}=0$. Similarly, we have fully-solved-out approximations of changes in sales and factor shares (to the first-order):

$$
\begin{aligned}
& \lambda_{i} \Delta \log \lambda_{i} \approx \theta_{0} \operatorname{Cov}_{\Omega^{(0)}}\left(\Delta \log \omega_{0}, \Psi_{(i)}\right) \\
&+\sum_{j \in 1+\mathcal{N}} \lambda_{j} \frac{\theta_{j}-1}{\theta_{j}} \operatorname{Cov}_{\Omega^{(j)}}\left(\sum_{k \in \mathcal{N}} \Psi_{(k)} \Delta \log A_{k}+\sum_{f \in \mathcal{G}} \Psi_{(f)} \Delta \log L_{f}, \Psi_{(i)}\right) .
\end{aligned}
$$




\section{A.4 General Expression for the Redeploying-Suppressed-Factors Ex- periment in Section 4.1.3}

The gains from reallocations with general shocks are given up to the second order by

$$
\begin{aligned}
\Delta \log \tilde{Y}- & \Delta \log Y \approx-\lambda_{h} \Delta \log L_{h}+\frac{1}{2} \lambda_{h}\left(1-\frac{\lambda_{h}}{\lambda_{h^{\prime}}}\right)\left(\Delta \log L_{h}\right)^{2} \\
+ & \sum_{j \in 1+\mathcal{N}} \frac{1}{2} \lambda_{j} \frac{\theta_{j}-1}{\theta_{j}} \operatorname{Var}_{\Omega^{(j)}}\left(\sum_{k \in \mathcal{N}} \Psi_{(k)} \Delta \log A_{k}+\sum_{f \in \mathcal{G}} \Psi_{(f)} \Delta \log \tilde{L}_{f}\right) \\
- & \sum_{j \in 1+\mathcal{N}} \frac{1}{2} \lambda_{j} \frac{\theta_{j}-1}{\theta_{j}} \operatorname{Var}_{\Omega^{(j)}}\left(\sum_{k \in \mathcal{N}} \Psi_{(k)} \Delta \log A_{k}+\sum_{f \in \mathcal{G}} \Psi_{(f)} \Delta \log L_{f}\right) \\
& -\frac{1}{2} \theta_{0} \operatorname{Cov}_{\Omega^{(0)}}\left(\Delta \log \omega_{0}, \Psi_{\left(h^{\prime}\right)} \frac{\lambda_{h}}{\lambda_{h^{\prime}}} \Delta \log L_{h}\right) .
\end{aligned}
$$

\section{A.5 Factor Supply à la Roy}

We now combine our baseline model with a labor supply block à la Roy.

\section{A.5.1 Extending the Model}

We use the Roy model of Galle et al. (2017). We assume that there are groups indexed by $h \in \mathcal{H}$, each of size $L^{h}$. Each group supplies one or many factors $f \in \mathcal{G}^{h}$, but that no factor is supplied by two groups. The wages of the different factors are $p_{f}$. The supply of each factor $f$ is essentially given by the equivalent of a CES production function for joint production. This production function also has a micro-foundation where a heterogeneous population of size $L^{h}$, each with one physical unit of labor, draws different efficiency units of labor in the different sectors $f \in \mathcal{G}^{h}$ according to independent Frechet distributions and chooses the sector where it earns the most.

We allow for tax wedges $\omega_{f}$ between perceived wages $p_{f} / \omega_{f}$ and true wages $p_{f}$. We normalize these tax wedges with $\sum_{g \in \mathcal{G}^{h}} \bar{\lambda}_{g}^{h} \omega_{g}=1$. The tax wedges are equal to one at the initial equilibrium $\left(\bar{\omega}_{f}=1\right)$. Shocks to the tax wedges are shocks to the composition of labor. They can represent changes in labor-supply preferences. For example, a tax wedge can open up ( $\left.\Delta \log \omega_{f}>0\right)$ because supplying one unit of labor $f$ puts an agent at risk of being infected by a virus (an internality). The tax wedges can also represent regulations by the government. For example, a tax wedge can open up because the government is worried that an agent supplying one unit of labor $f$ puts others at risk of being infected by the virus (an externality). Similarly, shocks to $L^{h}$, can represent either preference-driven 
or policy-induced changes in overall factor supply. For example, this could come from voluntary or mandated confinement of certain population groups based on demographics to avoid infection and contagion.

For both types of shocks to factor supplies, as in the baseline model, the distinction between preference-driven and government-induced is irrelevant for the equilibrium allocation and for real GDP or output. This distinction only becomes relevant for welfare, and we refer the reader to Appendix A.5.3 below for the corresponding analysis.

The supply the different types of labor is given by

$$
\frac{L_{f}}{\bar{L}_{f}}=\frac{L^{h}}{\bar{L}^{h}} \frac{\left(\frac{p_{f}}{\bar{p}_{f}} \frac{\bar{\omega}_{f}}{\omega_{f}}\right)^{\phi^{h}-1}}{\left(\sum_{g \in \mathcal{G}^{h}} \bar{\lambda}_{g}^{h}\left(\frac{p_{g}}{\bar{p}_{g}} \frac{\bar{\omega}_{g}}{\omega_{g}}\right)^{1+\phi^{h}}\right)^{\frac{\phi^{h}}{1+\phi^{h}}}},
$$

where $\lambda_{g}^{h}=\lambda_{g} / \lambda^{h}$ and $\lambda^{h}=\sum_{g \in \mathcal{G}^{h}} \lambda_{g}$, and where $\phi^{h}$ is the elasticity of substitution between factors. We also define $\lambda_{g}^{h}=0$ if $g \notin \mathcal{G}^{h}$.

Proposition 7. Changes in output can be approximated to the second order by

$$
\begin{aligned}
\Delta \log Y \approx \sum_{i \in 1+\mathcal{N}} & \lambda_{i} \Delta \log A_{i}+\sum_{h \in \mathcal{H}} \lambda^{h} \Delta \log L^{h} \\
+\frac{1}{2} \sum_{i \in 1+\mathcal{N}} \lambda_{i} \Delta \log \lambda_{i} \Delta & \log A_{i}+\frac{1}{2} \sum_{h \in \mathcal{H}} \lambda^{h} \Delta \log \lambda^{h} \Delta \log L^{h} \\
& +\frac{1}{2} \sum_{h \in \mathcal{H}} \lambda^{h} \sum_{f \in \mathcal{G}^{h}} \lambda_{f}^{h}\left(\Delta \log L_{f}-\Delta \log L^{h}\right) \Delta \log \omega_{f},
\end{aligned}
$$

where

$$
\begin{aligned}
& \frac{1}{2} \sum_{h \in \mathcal{H}} \lambda^{h} \sum_{f \in \mathcal{G}^{h}} \lambda_{f}^{h}\left(\Delta \log L_{f}-\Delta \log L^{h}\right) \Delta \log \omega_{f}= \\
& \frac{1}{2} \sum_{h \in \mathcal{H}} \lambda^{h} \frac{\phi^{h}}{1+\phi^{h}} \operatorname{Cov}_{\lambda^{h}}\left(\Delta \log \lambda_{f}, \Delta \log \omega_{f}\right)-\frac{1}{2} \sum_{h \in \mathcal{H}} \frac{\phi^{h}}{1+\phi^{h}} \lambda^{h} \operatorname{Var}_{\lambda^{h}}\left(\Delta \log \omega_{f}\right),
\end{aligned}
$$

and where changes in sales and factor shares $\Delta \log \lambda_{i}$ and $\Delta \log \lambda^{h} \approx \sum_{g \in \mathcal{G}^{h}} \lambda_{g}^{h} \Delta \log \lambda_{g}$ are given as function of microeconomic primitives by Corollary 1 combined with the factor supply equations $\Delta \log L_{f} \approx \Delta \log L^{h}+\left(\phi^{h} /\left(1+\phi^{h}\right)\right)\left(\left(\Delta \log \lambda_{f}-\Delta \log \omega_{f}\right)-\mathbb{E}_{\lambda^{h}}(\Delta \log \lambda-\Delta \log \omega)\right)$.

The first-order terms on the first line on the right-hand side of the first expression, as well as the second-order terms on the second line, are familiar from the previous analysis. Even though shocks to tax wedges do not directly appear in these terms, it is important to 
realize that they appear indirectly by impacting changes in sales and factor shares $\Delta \log \lambda_{i}$ and $\Delta \log \lambda^{h}$.

There are also new second-order terms on the third line of the first expression. These terms account for the impact of the shocks to the wedges over and above their impact on changes in sales and factor shares $\Delta \log \lambda_{i}$ and $\Delta \log \lambda^{h}$ on the second line. These new effects are intuitive. It is important to realize that output is not welfare. And from the perspective of output, tax wedges can be thought of as distortions. As explained above, this is true whether or not they represent shocks to preferences or government interventions. The reason is that they are not reflected in transacted prices and so their potential preference benefits are not picked up by real GDP or output. Because tax wedges act as distortions, they create misallocation, and the two new terms measure this misallocation as the sizes of Harberger triangles $(1 / 2) \sum_{h \in \mathcal{H}} \lambda^{h} \sum_{f \in \mathcal{G}^{h}} \lambda_{f}^{h}\left(\Delta \log L_{f}-\Delta \log L^{h}\right) \Delta \log \omega_{f}$. They reduce output if they discourage the supplies of factors that decrease in equilibrium.

These new terms can be rewritten using the second expression. They result in additional output reductions if tax wedges discourage the supply of factors with decreasing equilibrium shares as measured by $(1 / 2) \frac{1}{2} \sum_{h \in \mathcal{H}} \lambda^{h}\left(\phi^{h} /\left(1+\phi^{h}\right)\right) \operatorname{Cov}_{\lambda^{h}}\left(\Delta \log \lambda_{f}, \Delta \log \omega_{f}\right)$. They also result in additional output reductions if they are very dispersed as measured by $(1 / 2) \frac{1}{2} \sum_{h \in \mathcal{H}} \lambda^{h}\left(\phi^{h} /\left(1+\phi^{h}\right)\right) \operatorname{Var}_{\lambda^{h}}\left(\Delta \log \omega_{f}\right)$. Both effects are proportional with the elasticities of substitution in factor supply $\phi^{h} /\left(1+\phi^{h}\right)$.

\section{A.5.2 Illustrative Examples}

As a concrete example, imagine that one of the sectors is restaurants $(R)$ which is produced using a specific form of labor $(L R)$ and which sells only to final demand. Imagine that another of the sectors is online retail which is produced with an aggregate of two forms of labor $\left(L O R^{\prime}\right.$ ) and (LOR) with initial shares $\Omega_{O R, L O R^{\prime}}$ and $\Omega_{O R, L O R}$ and with an elasticity of substitution $\theta>1$. Labor $L O R^{\prime}$ is supplied inelastically and $L O R$ is supplied by the same group $(L R+L O R)$ as $L R$ with an elasticity $\phi$. Assume that all the elasticities of substitution in production and in final demand are equal to one except for that between $L O R$ and $L O R^{\prime}$ in the production of $O R$. Imagine that because of confinement measures a pandemic, preferences shift away from restaurants $\left(\Delta \log \omega_{0 R}<0\right)$ and towards online retail $\left(\Delta \log \omega_{0 O R}>0\right)$ and that there is a reduction $\left(\Delta \log L_{L R+L O R}<0\right)$ in the overall supply of workers supplying $L R$ and $L O R$. The impact of the shocks on output is given 
by

$$
\begin{aligned}
\Delta \log Y & \approx \lambda_{L_{L R+L O R}} \Delta \log L_{L R+L O R} \\
& +\frac{1}{2} \frac{\frac{\theta-1}{\theta} \lambda_{L O R}\left(1-\Omega_{O R, L O R}\right)}{1-\frac{\phi}{1+\phi} \frac{\theta-1}{\theta}\left(1-\lambda_{L O R}\right)\left(1-\Omega_{O R, L O R}\right)}\left(\Delta \log L_{L R+L O R}\right)^{2} \\
& +\frac{1}{2} \Delta \log L_{L R+L O R} \frac{\lambda_{L O R} \Delta \log \omega_{O O R}+\lambda_{R} \Delta \log \omega_{O R}\left(1-\frac{\phi}{1+\phi} \frac{\theta-1}{\theta}\left(1-\Omega_{O R, L O R}\right)\right)}{1-\frac{\phi}{1+\phi} \frac{\theta-1}{\theta}\left(1-\lambda_{L O R}\right)\left(1-\Omega_{O R, L O R}\right)} .
\end{aligned}
$$

The first-order impact of the shock on output is negative. In the absence of shocks to demand, the second-order terms mitigates the reduction in output, the more so, the higher is the elasticity of reallocation $\phi$, but even when there is no reallocation $(\phi=0)$. When there is no reallocation, this is because the share of $L O R$ decreases as $O R$ substitutes towards $L O R^{\prime}$. When there is reallocation $(\phi>0), L R+L O R$ reallocates towards $L R$ and away from $L O R$, and this further mitigates the reduction in output. When there are also shocks to demand, these reallocation patterns can be reversed so hat $L R+L O R$ reallocates towards $L O R$ and away from $L R$. A sufficient condition for the shock to be further mitigated is that the shock to demand redirects demand away from $L R+L O R$ $\left(\lambda_{L O R} \Delta \log \omega_{0 O R}+\lambda_{R} \Delta \log \omega_{0 R}<0\right)$.

\section{A.5.3 Welfare}

We proceed as in Appendix A.2. Changes in these tax wedges $\Delta \log \omega_{f}=\Delta \log \omega_{f}^{\text {pref }}+$ $\Delta \log \omega_{f}^{\text {pol }}$ reflect changes in preferences and policy interventions captured respectively by $\Delta \log \omega_{f}^{\text {pref }}$ and $\Delta \log \omega_{f}^{\text {pol }}$. We impose the normalizations

$$
\sum_{f \in \mathcal{G}} \bar{\lambda}_{f} \Delta \log \omega_{f}^{\text {pref }}=\sum_{f \in \mathcal{G}} \bar{\lambda}_{f} \Delta \log \omega_{f}^{\text {pol }}=0
$$

For simplicity, we assume that there are no shocks to demand. The results can easily be extended with shocks to demand by combining this analysis with that of Appendix A.2. We get 


$$
\begin{aligned}
\Delta \log W \approx \sum_{i \in \mathcal{N}-\mathcal{G}} & \lambda_{i} \Delta \log A_{i}+\sum_{h \in \mathcal{H}} \lambda^{h} \Delta \log L^{h} \\
& +\frac{1}{2} \sum_{i \in \mathcal{N}-\mathcal{G}} \lambda_{i} \Delta \log \lambda_{i} \Delta \log A_{i}+\frac{1}{2} \sum_{h \in \mathcal{H}} \lambda^{h} \Delta \log \lambda^{h} \Delta \log L^{h} \\
+ & \frac{1}{2} \sum_{h \in \mathcal{H}} \frac{\gamma_{h}-1}{\gamma_{h}} \sum_{f \in \mathcal{G}^{h}} \lambda_{f} \Delta \log \lambda_{f}\left(\Delta \log \omega_{f}^{\mathrm{pol}}-\Delta \log \omega_{f}^{\mathrm{pref}}\right) \\
& \frac{1}{2} \sum_{h \in \mathcal{H}} \frac{\gamma_{h}-1}{\gamma_{h}} \lambda^{h}\left(\operatorname{Var}_{\lambda^{h}}\left(\Delta \log \omega_{f}^{\mathrm{pref}}\right)-\operatorname{Var}_{\lambda^{h}}\left(\Delta \log \omega_{f}^{\mathrm{pol}}\right)\right) .
\end{aligned}
$$

